OPEN ACCESS

Edited by: Mike Martin,

University of Zurich, Switzerland

Reviewed by: Rachael Frost,

University College London,

United Kingdom

Arkers Kwan Ching Wong, Hong Kong Polytechnic University,

Hong Kong SAR, China

${ }^{*}$ Correspondence:

Lotte P. Brinkhof

I.p.brinkhof@uva.n

Specialty section:

This article was submitted to

Psychology of Aging,

a section of the journal

Frontiers in Psychology

Received: 03 August 2021

Accepted: 13 October 2021

Published: 15 November 2021

Citation:

Brinkhof LP, Huth KBS, Murre JMJ,

de Wit S, Krugers $H J$ and Ridderinkhof KR (2021) The Interplay

Between Quality of Life and

Resilience Factors in Later Life: A

Network Analysis.

Front. Psychol. 12:752564.

doi: 10.3389/fpsyg.2021.752564

\section{The Interplay Between Quality of Life and Resilience Factors in Later Life: A Network Analysis}

\author{
Lotte P. Brinkhof ${ }^{1,2,3 *}$, Karoline B. S. Huth ${ }^{1,2,4}$, Jaap M. J. Murre ${ }^{1,2,3}$, Sanne de Wit ${ }^{1,2,3}$, \\ Harm J. Krugers ${ }^{2,3,5}$ and K. Richard Ridderinkhof $f^{1,2,3}$
}

\begin{abstract}
'Department of Psychology, Faculty of Behavioural and Social Sciences, University of Amsterdam, Amsterdam, Netherlands, ${ }^{2}$ Centre for Urban Mental Health, University of Amsterdam, Amsterdam, Netherlands, ${ }^{3}$ Amsterdam Brain \& Cognition (ABC), University of Amsterdam, Amsterdam, Netherlands, ${ }^{4}$ Department of Psychiatry, Amsterdam University Medical Centre, Amsterdam, Netherlands, ${ }^{5}$ Faculty of Science, Swammerdam Institute for Life Sciences, University of Amsterdam, Amsterdam, Netherlands
\end{abstract}

Age-related challenges and transitions can have considerable social, psychological, and physical consequences that may lead to significant changes in quality of life (QoL). As such, maintaining high levels of QoL in later life may crucially depend on the ability to demonstrate resilience (i.e., successful adaptation to late-life challenges). The current study set out to explore the interplay between several resilience factors, and how these contribute to the realization and maintenance of (different facets of) QoL. Based on the previous work, we identified behavioral coping, positive appraisal, self-management ability, and physical activity as key resilience factors. Their interplay with (various facets of) QoL, as measured with the WHOQOL-OLD, was established through network analysis. In a sample of community-dwelling older adults $(55+; N=1,392)$, we found that QoL was most strongly (and directly) related to positive appraisal style and self-management ability. Among those, taking care of multifunctional resources (i.e., yielding various benefits at the same time) seemed to be crucial. It connected directly to "satisfaction with past, present, and future activities," a key facet of QoL with strong interconnections to other QoL facets. Our analysis also identified resilience factor(s) with the potential to promote QoL when targeted by training, intervention, or other experimental manipulation. The appropriate set of resilience factors to manipulate may depend on the goal and/or facet of QoL that one aims to improve.

Keywords: quality of life, resilience, older adults, network analysis, self-management ability, coping, positive appraisal, physical activity

\section{INTRODUCTION}

Aging gives rise to certain challenges and transitions that are often unavoidable (e.g., cognitive decline, physical deterioration, or loss of spouse and friends; Janssen et al., 2002; Newman et al., 2003; Glisky, 2007; Vasconcelos et al., 2016; Wang et al., 2020). These can have considerable social, psychological, or physical consequences (Fried et al., 2015; Valtorta et al., 2016; Jaul and Barron, 2017; Ohrnberger et al., 2017a,b). While some older adults will be able to compensate 
for these changes, others may lack the capacity to adequately self-regulate and manage them, increasing the risk to experience declines in quality of life (QoL; e.g., Hildon et al., 2010; Hayman et al., 2017). This study set out to explore how positive resources, or resilience factors, can contribute to the realization and maintenance of QoL in later life and thereby promote successful aging. A secondary aim was to identify those resilience factor(s) that hold potential to promote QoL when targeted by trainings, interventions, or other kinds of experimental manipulations, thereby gaining insight into how older adults' control of their own lives (or empowerment) could be enhanced.

While successful aging has commonly been defined in terms of physical health and functioning, we consider it as the process of developing and maintaining the functional ability and competence that enables well-being and QoL at old age (cf. World Health Organization's definition of healthy aging; WHO, 2020a). This suggests that the key to thriving in late life may be the ability to demonstrate resilience to age-related challenges and transitions (Greve and Staudinger, 2006; Harris, 2008; Netuveli and Blane, 2008; Fry and Keyes, 2010; MacLeod et al., 2016). Resilience can be defined as the process of successfully adapting to difficult or challenging life experiences, especially through mental, emotional, and behavioral flexibility and adjustment to external and internal demands (American Psychological Association, 2020). In late life, resilience can be conceptualized as a protective characteristic that promotes the maintenance of functional ability or competence, well-being, and QoL despite the presence of internal or external age-related threats, risks, or adversity (Ryff et al., 1998; Mehta et al., 2008; Hildon et al., 2010; Stephens et al., 2015; Hayman et al., 2017). While resilience has often been measured as a personality characteristic or trait (Windle et al., 2011), it has increasingly been evaluated in terms of potential health outcomes as well (e.g., QoL or well-being; Netuveli et al., 2008; Hildon et al., 2010; Chmitorz et al., 2018; Kalisch et al., 2020), characterized by multiple resilience factors. In the current study, we build on the latter outcome-based perspective by focusing on the interplay between such factors in relation to QoL specifically (see also Hildon et al., 2008, 2010). This approach acknowledges the complex and emergent nature of resilience (Brinkhof et al., 2021).

According to the World Health Organization, QoL can be defined as "individuals' perceptions of their position in life in the context of the culture and value systems in which they live and in relation to their goals, expectations, standards and concerns" (WHO, 1997). It refers to the cognitive appraisal of one's life, rather than the emotional response to it (which comprises the concept of well-being), and therefore extends beyond the mere assessment of health status and functioning (Upton and Upton, 2015). QoL is often used as an umbrella term in aging research to describe a number of outcomes that are believed to be important in the lives of older adults (Rejeski and Mihalko, 2001; Upton and Upton, 2015). Accordingly, it has been recognized as a pillar for pleasant living in later life (Ekerdt et al., 2017), and the maintenance of good QoL has been endorsed as an important focal point for governments and health policies (Bowling, 2007; WHO, 2020b). The most frequently used instrument to assess QoL in older adults is the World Health Organization Quality of Life-Older Adults Module (WHOQOL-OLD). This instrument recognizes the multidimensionality of QoL and comprises six facets that are considered especially important to older adults. These include sensory abilities (SAB); autonomy; satisfaction with past, present, and future activities and achievements in life; social participation (SOP); concerns, worries and fears about death and dying (DAD); and being able to have personal and intimate relationships.

Researchers have tried to ascertain the variety of resources that may help to shield individuals against challenges and setbacks or reduce their negative consequences (Clark et al., 2011) and have thus identified a wide range of resilience factors (e.g., Greve and Staudinger, 2006; Fontes and Neri, 2015; MacLeod et al., 2016). For the current study, we focused on a small selection of factors that appear particularly relevant in relation to QoL and its facets, and hold great potential for intervention. A factor that is widely studied is coping ability. Coping generally refers to the cognitive and behavioral efforts (e.g., emotion regulation and problem solving) implemented to modulate internal or external demands that are appraised as taxing or exceeding personal resources (Lazarus and Folkman, 1984; Endler and Parker, 1990; Garnefski et al., 2001). Adaptive coping skills are thought to be critical for adaptation at old age (Gamrowska and Steuden, 2014; MacLeod et al., 2016) and have been associated with higher QoL (Hildon et al., 2010; Rey and Extremera, 2016; León-Navarrete et al., 2017). Two recently developed instruments that capture various cognitive (emotion regulation) and behavioral coping strategies are the Positive Appraisal Style Scale (PASS) and the Behavioral Coping Scale (BCS). These scales were developed based on a factor analysis (see Kalisch et al., 2020) on the subscale level of the brief COPE (Carver, 1997), CERQ-short (Garnefski and Kraaij, 2006a), and two additional items on distanced stressor appraisal. The 14-item PASS reflects positive appraisal content and processes based on seven subscales (i.e., distanced stressor appraisal, positive reappraisal, acceptance, putting into perspective, refocus on planning, positive refocusing and humor). The 8-item BCS reflects less cognitive and more behavioral coping and includes four subscales: use of instrumental support, emotional support seeking, venting of emotions, and planning/acting out. It has been confirmed that both the PASS and BCS positively predict outcome-based resilience (i.e., General Health Questionnaire; see Kalisch et al., 2020; Veer et al., 2020). Moreover, some of the subcomponents of the scales have also been linked frequently to positive (mental) health outcomes (Garnefski et al., 2001; Kraaij et al., 2002; Garnefski and Kraaij, 2006b; Raut et al., 2014; Nowlan et al., 2015) and have been shown to be modifiable (Garland et al., 2009; Hanley and Garland, 2014; Satorres et al., 2018). Yet, the exact relationships with QoL have not been investigated.

Although studied less thoroughly, and not directly related to resilience in previous research, self-management ability (SMA) may also comprise an important factor that underpins resilience in old age (Schuurmans et al., 2004; Cramm et al., 2014). It has been suggested that successful aging, and the realization 
and maintenance of well-being in particular, depends on the proactive management of external resources (social and physical, e.g., friends and physical fitness; Steverink et al., 2005), that often decline with age. Within the context of the Self-Management of Well-being theory, Steverink et al. (2005) identified six SMAs that are important to adequately manage one's life and external resources in the process of aging (Nieboer and Lindenberg, 2002; Steverink et al., 2005; Cramm et al., 2012a). These include the ability to take initiative; invest in resources for long-term benefits; be self-efficacious with regard to managing resources; keep a positive frame of mind regarding the future; maintain care of a variety of resources (providing a buffer in case a resource is lost); and maintain multifunctional resources (i.e., yielding various benefits at the same time: killing two birds with one stone). An example of a multifunctional resource is a friend/spouse, who can satisfy one's need for affection, but also supports the fulfilment of other needs (e.g., sharing burdens or stimulation, by jointly participating in interesting activities). All facets were identified under the assumption that they should in principle be modifiable by training, interventions, or other kinds of manipulations (e.g., support of community nurses) to improve health outcomes, which has subsequently been confirmed in several studies (e.g., Schuurmans, 2004; Frieswijk et al., 2006; Kremers et al., 2006; Cramm and Nieboer, 2017). Unlike the fundamental and theoretical relationship with wellbeing, the role of self-management and how the orchestrated use of these abilities may help to sustain QoL in later life is relatively underexplored (e.g., Cramm and Nieboer, 2017).

In addition to these psychosocial factors, being physically active has also been identified as an important factor that could promote resilience (e.g., Childs and de Wit, 2014). Besides improving (physical) health (e.g., Arroll and Beaglehole, 1992; Vella et al., 2001; Taylor, 2014), physical activity has also been related to a reduced incidence of mental health problems (Goodwin, 2003). Moreover, physical activity interventions have been shown to improve mental well-being and QoL reports (Conn et al., 2009; Windle et al., 2010; Figueira et al., 2012). The practice of physical activity by older adults may also strengthen their abilities to manage or cope with challenges or difficulties (Ho et al., 2015; Ávila et al., 2018) and interact with factors such as coping, appraisal, and self-management, thereby indirectly promoting QoL. On the other hand, it has also been proposed that strengthening resilience may improve the adherence to exercise behaviors (e.g., Resnick and Inguito, 2011). Surprisingly, the number of studies investigating such relationships, especially among older adults, is scarce. More studies are needed to understand how exactly being physically active plays a role in building resilience at old age (Rejeski and Mihalko, 2001).

In the current study, we explored the interplay between behavioral coping (BC), positive appraisal style, SMA, and physical activity (PHY), and how these affect (the facets of) QoL. While Gerino et al. (2017) have also looked at the interplay between QoL and multiple psychosocial factors, including general self-efficacy and personality characteristics that are thought to enhance individual adaptation, we aim to build upon recent findings and explore the (relative) contribution of the aforementioned factors. Moreover, we will employ the WHOQOL-OLD, and the individual facets of that construct, which is more tailored to older adults than the WHOQOLBREF used by Gerino et al. (2017).

In order to obtain a comprehensive view of these interactions, we performed several network analyses in a sample of healthy, community-dwelling older adults ( $N=1,392,55$ years and above). This allowed us to visualize the interplay between (facets of) QoL and the aforementioned resilience factors. Of note, the aim of this study was not to characterize resilience in terms of stability of the network (e.g., weakly connected networks are considered more stable/resilient; see, e.g., Borsboom, 2017; Kalisch et al., 2019), but rather to focus on the interplay between QoL as an outcome of resilience and some factors that are thought to underpin resilience in later life. Specifically, we sought to determine the relative strengths of the contributions of the empirically supported resilience factors to overall QoL, as well as to the six individual facets. Given the novelty of using a complex system approach to elaborate on the interplay between resilience factors, and how they in turn affect QoL, we had no strong a priori hypotheses on the interrelations. Nevertheless, we expected that all factors would be positively related to (different facets of) QoL, either directly or indirectly. This allowed us to also assess which factors might hold potential for modulating QoL through intervention, by examining the patterns and strengths of the relationships, as well as their relative importance as predictors of QoL within the network. Unlike previous studies that have looked at various resilience factors in isolation from each other (see MacLeod et al., 2016), our approach can widen our understanding of how resilience in later life emerges out of the interactions between some of its underlying components. Unsuspected pathways (e.g., mediation effects) through which QoL in later life could potentially be realized and maintained may be revealed accordingly. Hence, the current study can provide unique insights on how certain characteristics can contribute to higher levels of resilience in later life.

\section{MATERIALS AND METHODS}

\section{Sample Characteristics}

Data are derived from an ongoing large-scale online study on successful aging and resilience (Brinkhof et al., 2021; approved by the local ethics committee of the University of Amsterdam, 2020-DP-12556), where participants complete an inventory including several questionnaires and tests that cover a multitude of relevant factors from multiple domains (e.g., physical, psychological, cognitive, social, and environmental). It took approximately $1-1.5 \mathrm{~h}$ to complete the entire inventory, but participation could be divided over several days. Participants were eligible to enroll in this online inventory when they were 55 years or older, living in the Netherlands and had no Alzheimer's Disease, vascular dementia, frontotemporal dementia, Lewy body dementia, or other dementia diagnosis. While (apparent) age-related declines may generally be expected at a somewhat older age, some individuals already experience gradual declines 
in physical or mental capacity at a younger age. Hence, a relatively broad age range was adopted. Other exclusion criteria were insufficient command of the Dutch language, impaired vision, or not being able to perform the operations required to successfully use a computer or laptop independently (i.e., mouse clicks, pressing keys on the keyboard). Participants were forced to answer each question in order to proceed through the inventory. The first 1,500 participants that completed the entire inventory within 14 days after they started with the first part of the study were included in the current study. These participants took part in the study between October 5, 2020, and January 11, 2021, in the midst of the COVID-19 pandemic.

\section{Materials}

All materials included in the inventory are reported and described in (Brinkhof et al., 2021). The variables included in the current study are briefly repeated here.

\section{Quality of Life}

The Dutch language version of the WHOQOL-OLD (Power et al., 2005; Gobbens and van Assen, 2016) was used as measure of QoL. The measure comprises 24 items divided into six subscales of four items each: (1) sensory abilities (SAB; e.g., "To what extent do impairments to your senses (e.g., hearing, vision, taste, smell, touch) affect your daily life?"), (2) autonomy (AUT; e.g., "How much freedom do you have to make your own decisions?"), (3) past, present and future activities (PPF; e.g., "How satisfied are you with what you have achieved in life?”), (4) SOP (e.g., "To what extent do you feel that you have enough to do each day?"), (5) DAD (e.g., "How scared are you of dying?"), and (6) intimacy (INT; e.g., "To what extent do you feel a sense of companionship in your life?"). Responses were scored on 5-point Likert scales, with different wording, and summed across each subscale, as well as to a total QoL score. Higher scores (per scale) indicated better QoL. All items corresponding to the fifth subscale and some of items of the first and second subscale were reverse scored prior to summation.

\section{Positive Appraisal Style and Behavioral Coping}

The PASS and BCS were used to measure PAS and BC, respectively. While the BCS only constitutes items from the brief COPE, the PASS includes subscales from both the brief COPE, CERQ short and the two self-generated items (on distanced stressor appraisal). For that reason, participants were asked to fill out the "shortened COPE," including all subscales from the BCS and the humor subscale from the PASS, and the "shortened CERQ," including all remaining six subscales of the PASS, of which one constitutes the two self-generated items. All 10 items of the shortened COPE (including, e.g., "I've been getting emotional support from others") were scored on a 4 -point Likert Scale ( $1=$ not at all, $2=$ a little bit, $3=$ quite a lot, $4=\mathrm{a}$ lot) and 12-items of the shortened CERQ (e.g., "I think that I have to accept that this has happened") were scored on a 5-point Likert scale [ $1=$ (almost) never, $2=$ sometimes, $3=$ regularly, $4=$ often, $5=$ (almost) always]. The items from the subscales of the BCS were summed to a total BCS score of
8-32. The PASS score was determined by taking the average of the $z$-normalized scores of the items derived from the shortened COPE, the CERQ, and the self-generated items.

\section{Self-Management Ability}

The Self-Management Ability Scale (SMAS)-18 was used to measure SMA (Cramm et al., 2012b). The 18-item version of the original 30-item SMAS (Schuurmans et al., 2005) consists of six 3-item subscales. Items corresponding to the taking initiative (INI; e.g., "How often do you take initiative to get in touch with people who are dear to you?"), investing (INV; e.g., "Do you ensure that you have enough interests on a regular basis [such as a hobby] to keep you active?"), and positive frame of mind (PFM; e.g., "When you have a bad day, how often do you think that things will be better tomorrow?") subscales were scored on a 6-point Likert scale ranging from never (1) to very often (6). Another 6-point scale, with $1=$ none, $2=$ one, $3=$ two, $4=$ three or four, $5=$ five or six, and $6=$ more than six, was used to score the items corresponding to variety subscale (VAR; e.g., "How many hobbies or activities do you have on a regular basis?"). Items corresponding to the multifunctionality subscale (MUL; e.g., "The activities I enjoy, I do together with others.") were scored on a 5-point Likert scale ranging from strongly disagree (1) to strongly agree (5). Finally, items corresponding to the self-efficacy subscale (SEF; e.g., "Are you able to have friendly contacts with others?") were scored on a 5-point Likert scale ranging from I am certain that I cannot (0) to I am completely certain that I can (5). Scores were calculated by recoding the scores to $0-5$ or $0-4$ (for the 6 and 5-point scales, respectively) and multiplying the items with six options by 4 and the items with five options by 5 . After that, subscale scores were determined by taking the average of all items corresponding to each scale and multiplying that score by 5 . Hence, subscale scores range from 0 to 100 , with higher scores reflecting higher SMA in that dimension. SMAS total scores were calculated by taking the average of all mean subscale scores. Here, higher scores indicated higher overall SMA.

\section{Physical Activity}

While there are plenty of validated self-report questionnaires on physical activity, most of them are detailed and take a long time to complete. Since we aimed to minimize the burden of participants, we only used a limited number of questions to determine a general measure of physical activity. These were (partially) inspired by the Physical Activity Questionnaire used in the Longitudinal Aging Study Amsterdam (LASA Team, 2020). First, participants were asked to report whether they were sitting in a wheelchair or not. Subsequently, they had to indicate how many hours/min they have been doing light to moderate physical exertion (e.g., walking, cycling, and light household chores) in the past week. Similarly, they were asked to indicate how many hours/min they have been doing vigorous physical exertion (e.g., causing rapid breathing or shortness of breath; making you sweat, such as an intense bike ride, gymnastics/fitness, heavy household chores, etc.) in the past 
week. An intensity-weighted total physical activity score was subsequently calculated by taking into account the different intensities, using Metabolic Equivalent of Task (MET) scores (with one MET unit reflecting $1 \mathrm{kcal}$ per $\mathrm{kg}$ body weight per hour). In principle, each activity can be linked to a specific MET score, where energy consumption of light to moderate physical activities ranges from 1.6 to 5.9 METs, and energy consumption of vigorous physical activity is 6.0 MET or more. Here, we used an average MET score of 3.75 for mild to moderate and six for vigorous activities and multiplied those scores by the durations of the two intensity categories. Finally, these scores were summed and corrected (multiplied by) for sitting in a wheelchair or not, with no $=1$, normal wheelchair $=0.8$, mechanic wheelchair $=0.5$, and referred to as PHY.

\section{Stringency Index}

A measure of strictness of "lockdown style" policies that restricted people's behavior in the Netherlands at the time of participation was added to control for possible effects of the COVID-19 pandemic on QoL and the included resilience factors. The Stringency Index (SI) of the Oxford COVID-19: Government Response Tracker (OxCGRT) ${ }^{1}$ is a composite measure based on one health system policies indicator (i.e., presence of public info campaigns) and eight containment and closure policies indicators (i.e., closings of schools and universities, closings of workplaces, cancellations of public events, limits on private gatherings, closing of public transport, order to confine to the home, restrictions on internal movement between cities/regions, restrictions on international travel). If participation was divided over multiple days, the average SI of those days was used.

\section{Data Analysis \\ Overview}

\section{Main Analyses}

Several networks were estimated to explore the interplay between resilience factors and (facets of) QoL. Each network comprised the graphical representation of the relationships (i.e., edges) between the included variables (i.e., nodes). The first set of analyses included the following variables: overall QoL, BC, PAS, SMA, PHY, and Stringency Index (SI). To identify which resilience factors had the strongest contribution on overall QoL reports and could potential improve QoL when being manipulated, both a Gaussian graphical model (GGM) and directed relative importance network were constructed (six nodes). While the former provided us with information on the unique relationships between the nodes, the latter particularly enabled us to explore the potential causal relationships among the individual nodes. The edges in the GGM are undirected and specify the strength of association (positive or negative) between two nodes while taking into account all other nodes (i.e., partial correlations). In the relative importance network, edges are directed and represent the unique contribution of

${ }^{1}$ https://www.bsg.ox.ac.uk/research/research-projects/coronavirus-governmentresponse-tracker a given node - the regressor - to the prediction of another node - the outcome. The second set of analyses was conducted to establish how the resilience factors interact with the different facets of QoL, and how each factor may promote a specific set of facets. To this end, QoL was split into SAB, AUT, PFF, SOP, INT, and DAD, and similar networks were estimated (11 nodes).

\section{Exploratory Analyses}

A more exploratory set of analyses was performed by including all the individual facets of the SMAS (i.e., INT, INV, SEF, VAR, MUL, and PFM), in addition to all the other nodes included in the previous networks (15 nodes). Again, we constructed a GGM and directed relative importance network and identified the interplay among those factors.

\section{Statistical Analysis \\ GGM Estimation}

The ggmModSelect algorithm, as included in the qgraph and bootnet R-packages (Epskamp et al., 2012, 2018a), was used to estimate unregularized GGMs. This algorithm searches for an optimal unregularized GGM, by minimizing the (extended) Bayesian information criterion (EBIC; Foygel and Drton, 2010; Isvoranu and Epskamp, 2021). This resulted in non-biased parameters, with the edges representing partial correlation coefficients. In our networks, EBIC hypertuning parameter $(\gamma)$ was set to 0 , indicating BIC model selection.

Interactions between factors were visualized and interpreted using qgraph (Epskamp et al., 2012), where nodes with stronger associations were positioned at the center of the network and nodes with weaker connections were placed in the periphery, according to the Fruchterman and Reingold (1991) algorithm. The thickness and saturation of the edges signify the magnitude of the partial correlation coefficients, where green and red edges represent positive and negative correlations, respectively. The stability of the edge weights was assessed by using nonparametric bootstrapping (i.e., resampling data with replacement, 1,000 samples; Efron, 1979; Bollen and Stine, 1992; Hastie et al., 2015) and constructing quantile intervals around the edge weights. The narrower the quantile intervals are, the more stable the edge-weight estimates. Second, a bootstrapped difference test was performed to identify whether edge weights significantly differed from each other. This was done by taking the difference between bootstrapped values of the edge weight and constructing a quantile interval around each difference score. Edges were deemed significantly different $(p<0.05)$ if zero was not in the quantile interval.

\section{Relative Importance Network Estimation}

To explore potential causal relationships among QoL (facets) and resilience factors, the structure of each GGM was also imposed on a relative importance network estimation. Using the $\operatorname{lgm}$ metric as provided by the relaimpo $\mathrm{R}$ software package (Grömping, 2006) and qgraph (Epskamp et al., 2012), we constructed several relative importance networks (Robinaugh et al., 2014; McNally et al., 2015; Heeren and McNally, 2016; 
Hoorelbeke et al., 2016; Bernstein et al., 2017). Relative importance was quantified as the proportional contribution in predictability (i.e., amount of explained variance, $R^{2}$ ) attributable to each predictor, ranging from 0 to 1 . The resulting networks consisted of two opposing directed edges between each pair of nodes, with the directionality of these edges representing the directionality of the predictions. Through this, we could distinguish the outstrength (i.e., the extent to which a certain node predicts the variance of the connected nodes) and instrength (i.e., the extent to which variance of a node is predicted by other connected nodes) of each node. We calculated the total out- and instrength for each node (i.e., sum, considering all relationships) and additionally only the direct relationships of the resilience factors with the QoL nodes(s) were considered. This enabled us to better explore the predictive strength (i.e., relative importance as predictor) of each node in influencing the network, as well as how easily a node might be changed by manipulating the nodes directly related to that node.

The stability of the directed edges of the relative importance network was assessed by using nonparametric bootstrapping, with quantile intervals being estimated for each directed edge. Subsequently, a quantile interval was estimated around the summed out- and instrength differences of interest (see above). A bootstrapped difference test was performed to identify significant differences $(p<0.05)$ between the two directed edges of each node-pair, as well as the summed out- and instrength differences of interest per node.

Finally, we established the stability of the directed edges under case-dropping subset bootstrapping. This allowed us to quantify stability in terms of a correlation stability coefficient (CS), the maximum proportion of cases that can be dropped to retain with $95 \%$ certainty a correlation of at least 0.7 with the original out and instrength estimations (Cohen, 1977). The higher the CS, the more stable the corresponding strength value.

\section{RESULTS}

\section{Descriptive Statistics}

Visual inspection revealed normal distributions for QoL, BC, PAS, and SMA, but a considerably skewed distribution of the PHY data. Hence, this variable was subjected to the robust median absolute deviation outlier detection mechanism (Leys et al., 2013), with a threshold of 2.5, leaving 1,392 participants (520 males, 871 females, one other) that were included in the analyses. Participants had a mean age of 68.7 years old (SD: 6.92, range 55-93), and only two of them used a mechanical wheelchair. Participants reported a considerably high educational attainment, with the majority $(86 \%, N=1,196)$ having completed a high education level (Verhage 6 or 7; Verhage, 1964).

Descriptive statistics of the QoL and the resilience factors of interest are reported in Table 1. Graphical results of the (main) stability analyses of the individual (directed) edges can be found in the supplemental results (Supplementary Figures 1-10). In general, the edge weights exhibited relatively small quantile intervals, but some of the absolute edge weights did not differ significantly from one
TABLE 1 | Descriptive statistics of the QoL (facets) and the resilience factors for all participants.

\begin{tabular}{lcc}
\hline Construct (abbreviation; possible range) & M (SD) & $\begin{array}{c}\text { Observed } \\
\text { range }\end{array}$ \\
\hline Quality of life (QoL; 24-100) & $94.6(9.52)$ & $51-120$ \\
Sensory abilities (SAB; 4-20) & $17.6(2.61)$ & $6-20$ \\
Autonomy (AUT; 4-20) & $15.9(2.00)$ & $7-20$ \\
Past, present, and future activities (PPF; 4-20) & $15.9(2.07)$ & $6-20$ \\
Social participation (SOP; 4-20) & $15.7(2.61)$ & $5-20$ \\
Death and dying (DAD; 4-20) & $14.7(2.89)$ & $4-20$ \\
Intimacy (INT; 4-20) & $14.9(2.92)$ & $4-20$ \\
Behavioral coping (BC; 8-32) & $21.5(3.71)$ & $9-32$ \\
Positive appraisal Style (PAS) & $0.03(0.60)$ & $-1.78-1.72$ \\
Self-management ability (SMA; 0-100) & $69.2(11.6)$ & $28.9-98.9$ \\
Taking initiative (INI; 0-100) & $69.7(16.7)$ & $20-100$ \\
Investment behavior (INV; 0-100) & $77.0(15.6)$ & $20-100$ \\
Self-efficacy (SEF; 0-100) & $58.6(16.14)$ & $6.67-100$ \\
Variety (VAR; 0-100) & $60.8(16.5)$ & $6.67-100$ \\
Multifunctionality (MUL; 0-100) & $83.2(14.9)$ & $25-100$ \\
Positive frame of mind (PFM; 0-100) & $66.0(17.3)$ & $0-100$ \\
Physical activity (PHY) & $3,641.6$ & $0-9,180$ \\
& $(2,109.6)$ & \\
Stringency Index (SI; 0-100) & $65.9(8.4)$ & $56.48-82.41$ \\
& &
\end{tabular}

${ }^{a} \mathrm{z}$-score.

${ }^{b}$ Composite score: physical activity duration weighted by MET.

another, indicating that the relative order and contribution (in case of outstrength vs. instrength comparisons) of some edges should be interpreted with caution. The CSs of both the in-and outstrength value were 0.75 for all estimated networks.

\section{Main Analyses \\ Which Resilience Factor Has the Strongest Contribution to Overall QoL?}

The first GGM highlighted that all nodes were directly or indirectly connected to each other, except for SI (Figure 1A). This indicates that the Stringency Index did not influence QoL, nor any other factors. QoL was directly connected to PAS and SMA, with the latter being positioned at the center of the network and showing the strongest relationship with QoL $\quad(r=0.39 \quad$ vs. $\quad r=0.15 \quad$ of PAS, $p<0.05$; Supplementary Figures 1, 2). Moreover, the relationships of both BC $(r=0.31)$ and PAS $(r=0.29)$ with SMA, as well as their own unique association $(r=0.14)$, were fairly strong. The partial correlation between SMA and PHY $(r=0.18)$ also indicated an indirect relationship between QoL and PHY. Finally, contrary to our expectations, $\mathrm{BC}$ was negatively related to PHY $(r=-0.09)$, although relatively weakly (all $p$ 's $<0.05$ ) with the edge set to zero relatively often (35\%) compared with all other edges $(<1 \%)$ with bootstrapping (Supplementary Figures 1, 2).

The relative importance network revealed that SMA and PAS together accounted for $27.8 \%$ of the variance in QoL (instrength), while QoL only explained 23.3\% (outstrength) of the variance of these resilience factors (Figure 1B; see Figures 2A,C for difference plots; $p<0.05$ ). SMA appeared to be the main hub in the network, exerting a large influence on all the other nodes (52.4\%). The total outstrength value was even significantly larger than the total instrength value $(42.9 \%, p<0.05$; Figures $2 A, C$; 
A

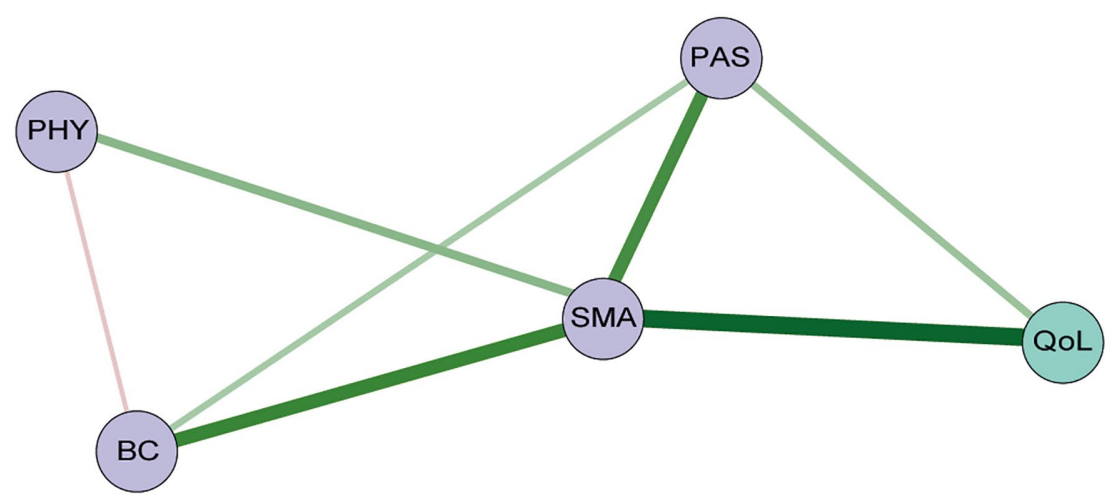

Quality of Life

O QOL: WHOQOL-OLD

Resilience Factors

SI $O$ BC: Behavioural Coping Scale

Maximum: 0.39

O PAS: Positive Appraisal Style Scale

O SMA: Self-Management Ability Scale

O PHY: Physical Activity

COVID-19

OSI: Stringency Index

B

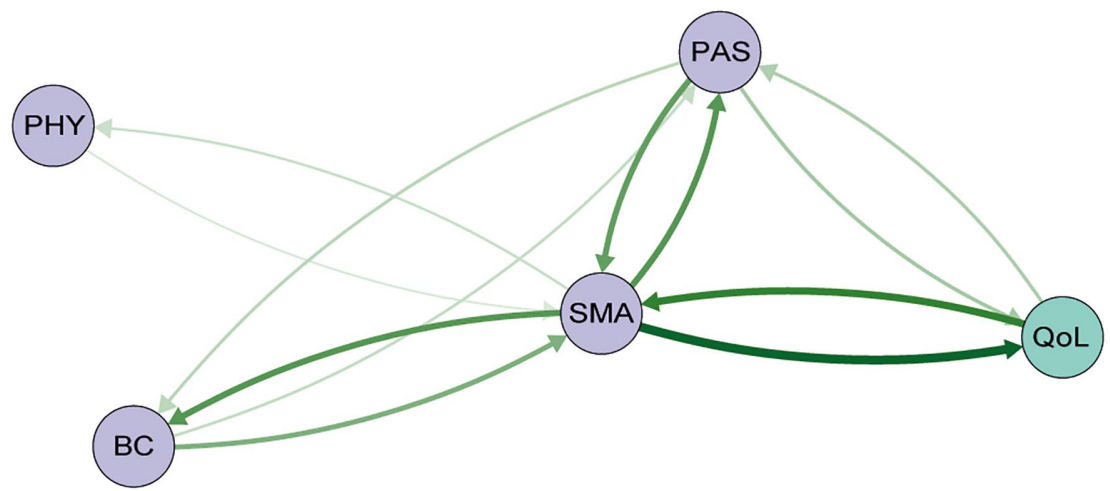

Maximum: 0.21

FIGURE 1 | Gaussian graphical model (GGM; A) and directed relative importance network (B) of overall quality of life (QoL; green), the resilience factors (purple), and the stringency index (blue). The maximum value represents the highest edge weight included in the network.

see Supplementary Figures 3, 4 for individual edges). PAS had the second largest outstrength value (25.2\%), but this was relatively similar to its instrength value $(24.7 \%, p>0.05)$. These results remained present after excluding the outstrength of SMA and PAS on the other resilience factors (Figures 2B,D). Finally, we observed a larger instrength than outstrength value of both $\mathrm{BC}$ and PHY (Figures 2A,C), suggesting that SMA (and PAS, in case of $\mathrm{BC}$ ) exerted a stronger influence on those factors than vice versa (both $p$ 's $<0.05$; and see Supplementary Figures 3, 4 for individual edges).

Hence, these networks suggest that manipulating SMA or PAS could result in improved QoL reports. Since SMA had the highest relative importance as a predictor of QoL (compared with PAS, $p<0.05$, Supplementary Figure 4), which was significantly higher than the corresponding instrength value, this resilience factor seemed to be most opportune to direct 
A

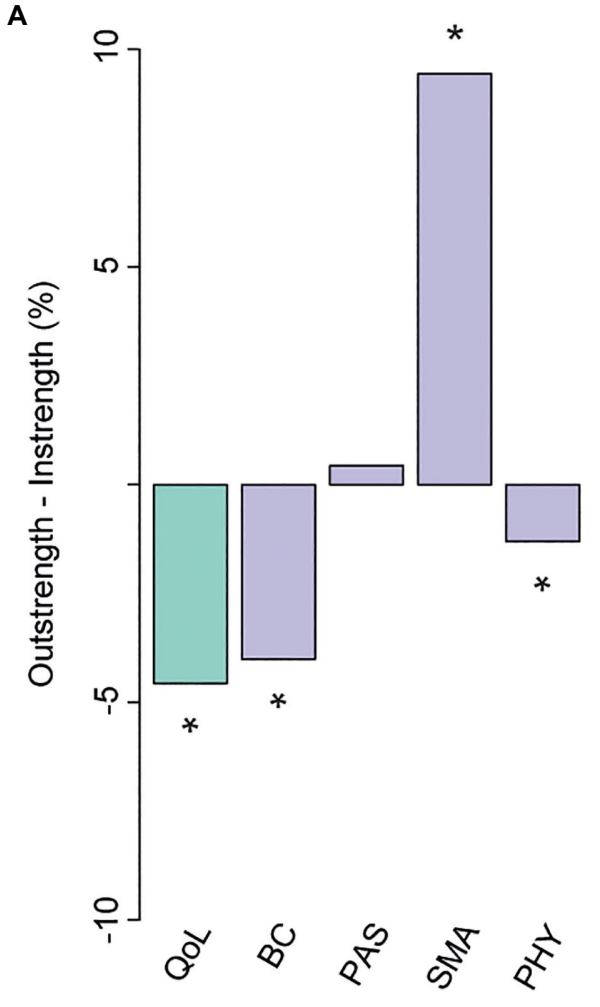

C

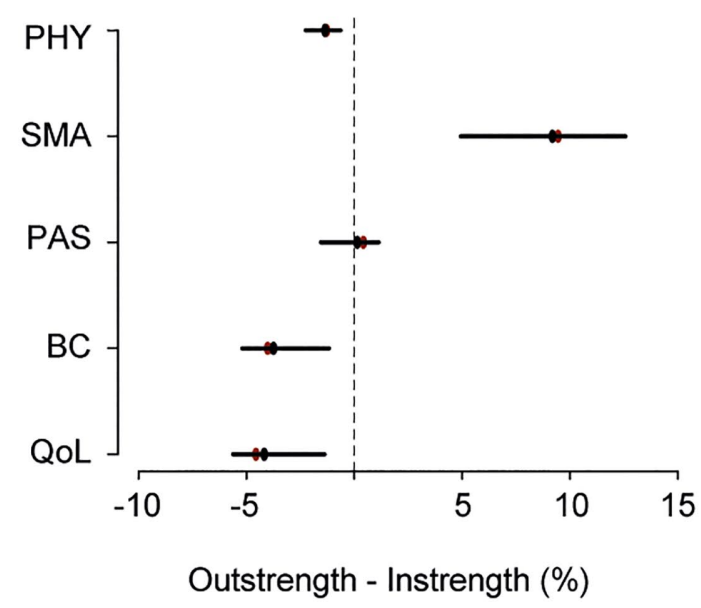

B

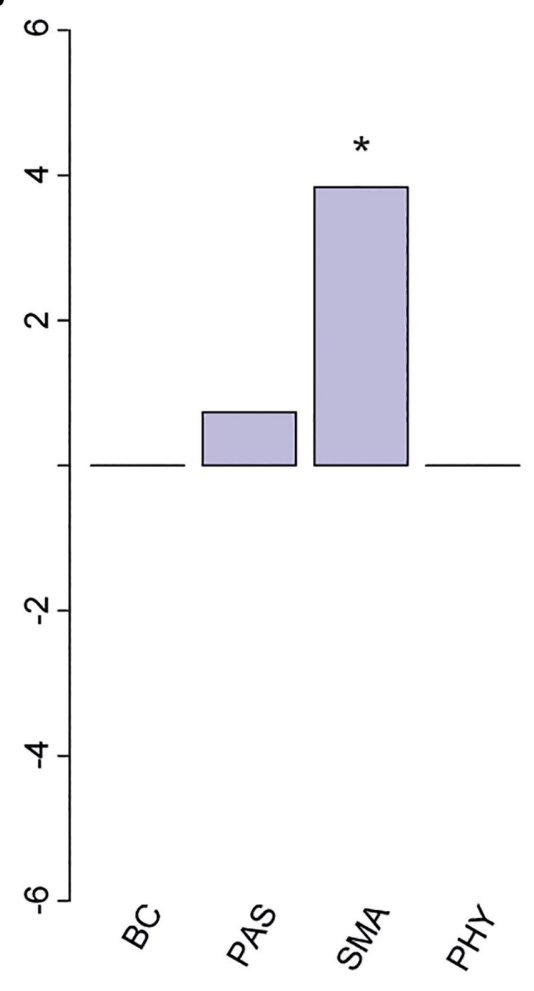

D

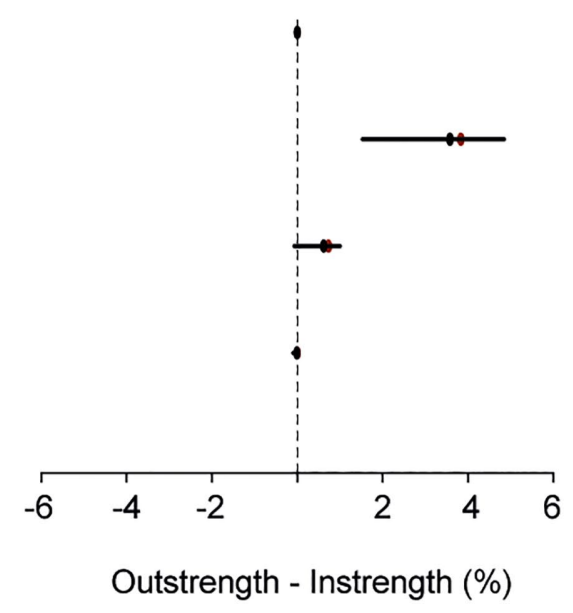

FIGURE 2 | The difference between overall outstrength and instrength of the nodes in the primary network (A) and the difference in outstrength and instrength of the relationships between the resilience factors and QoL only (B). Colors of the bar charts correspond to the nodes in the network in Figure 1. In plots (C,D), the bootstrapped mean is depicted in black and the sample mean in red. ${ }^{*} p<0.05$; nodes with quantile intervals containing zero are deemed to have an insignificant instrength and outstrength difference.

interventions or individual efforts on when trying to improve overall levels of QoL.

\section{How Are the Resilience Factors Related to Different Facets of QoL in Older Adults?}

The GGM with the individual facets of QoL being considered separately also showed that only the SI was not (in)directly connected to the other nodes (Figure 3A). As expected, we found similar relationships between PAS, SMA, BC, and
PHY compared with the overall QoL network (Supplementary Table 1). Edge weights of the relationships among all facets of QoL are shown in Supplementary Table 2. In addition to those clustered connections, some interesting relationships between the resilience factors and the individual facets of QoL were found (see Supplementary Table 3 for edge weights). SMA appeared to be especially strongly connected to the SOP facet of QoL and to a lesser extent to SAB, INT, and AUT (all lower than SOP: $p$ 's $<0.05$; see 
Supplementary Figures 5, 6). A direct relationship between SMA and both DAD and PPF was not observed, but PAS appeared to be related to both facets (with similar strengths, $p>0.05$ ). Finally, while $\mathrm{BC}$ was not directly related to overall QoL in the primary network, a relationship with the INT facet specifically was observed in this network (similar to SMA - INT, $p>0.05$, but more stable: $7 \%$ vs. $17 \%$ of bootstraps set to zero; Supplementary Figures 5, 6). This reveals that the QoL facets are directly related to different resilience factors.

In line with earlier findings, the directed relative importance network (Figure 3B) revealed that SMA had a relatively high total outstrength value $(57.1 \%)$ compared with its instrength value $(45.2 \%, p<0.05$; Figures $4 \mathrm{~A}, \mathrm{C}$; see Supplementary Figures 7, 8 for individual edges). PAS seemed to have a relatively larger instrength $(27.0 \%)$ than outstrength value $(25.5 \%)$, although this was not significant $(p>0.05)$. Subsequent calculations that only included the relationships with the QoL facets, and not with other resilience factors, revealed that SMA again exerted a larger total influence on the facets of QoL (27.5\%) than vice versa (21.1\%), although not significantly so, due to a relatively unstable estimate of the difference (large quantile interval; Figures 4B,D). For $\mathrm{BC}$, the total outstrength value was lower than the instrength value $(2.9 \%$ vs. $3.4 \%, p<0.05)$, whereas for PAS no difference was observed $(8.6 \%$ vs. $9.0 \%, p>0.05)$. Indeed, both the total instrength -and outstrength value of PFF were considerably high, with the latter in particular (59.7 vs. $91.0 \%, p<0.05)$. However, when excluding the relationships of PPF with the other QoL facets, the outstrength value (6.0\%) was relatively similar to the instrength value (5.1\%; $p>0.05$; Figures 4B,D). This suggest that PPF is an important facet of QoL, as it impacted many other QoL facets, but that PPF and PAS have similar relative importance as a predictor.

Thus, while overall QoL reports (first set of analyses) can possibly be enhanced by directing interventions or individual efforts on SMA, and PAS to a lesser extent, the second set of analyses further revealed that the selection of the most appropriate resilience factor (partially) depends on the goal. That is, boosting SMA could potentially result in improved reports on the SAB, SOP, INT, and AUT subscales. However, intervening on PAS may be particularly relevant when aiming to decrease worries about DAD or improve satisfaction about achievements in life and at things to look forward to (PPF).

\section{Exploratory Analyses}

How Do Different SMAs Relate to the QoL Facets and Other Resilience Factors?

While earlier analyses suggest that especially SMA is an important factor, it remains unclear what specific selfmanagement abilities are crucially involved. Through exploratory analyses, we aimed to establish whether there are substantial differences in the importance of the six SMA facets included in the SMAS. A third GGM again highlighted that almost all nodes were (in)directly connected to each other and revealed similar associations between the QoL facets (Figure 5A; Supplementary Table 4). Edge weights of connections among nodes of the individual facets of the
SMAS, as well as the other resilience factors, are reported in Supplementary Table 5. Not surprisingly, we observed a particularly strong connection between PAS and the PFM facet of the SMAS, and a relatively weak (and less stable) connection between PAS and VAR $(p<0.05$, Figure 5A; Supplementary Figures 9, 10). Both relationships had similar instrength and outstrength values ( $p$ 's $>0.05$; Figure 5B) and influenced each other equally. SEF was directly related to PHY, with SEF exerting a larger influence on PHY than vice versa $(4.7 \%$ vs. $2.6 \% ; p<0.05$; Figures $6 \mathbf{A}, \mathbf{C})$. This builds on the relationship between SMA and PHY observed in the previous networks and suggests that physical activity may indirectly enhance QoL, through increased self-efficacy, but that increasing self-efficacy specifically may also strongly (and even to a larger extent than vice versa) boost physical activity. In addition to the previously established connection with PAS, BC was also directly related to the SEF (not consistently, $30.6 \%$ of the bootstraps set to zero), INI, and MUL facets of the SMAS in this network. Taking all these edges together, the total instrength value of $\mathrm{BC}$ was higher compared with the outstrength value $(19.0 \%$ vs. $15.2 \%, p>0.05)$. This is in line with earlier suggestions that boosting (specific) SMAs may not only improve QoL, but also other resilience factors (e.g., BC or PHY) that can indirectly further enhance QoL.

Of all the SMAS facets, MUL had the most direct connections with facets of QoL (i.e., SAB, SOP, INT, and PPF; Figure 5A and Supplementary Table 6) and the largest total outstrengthinstrength difference (69.7-49.8\%; $p<0.05$; Figures 5B, 6A,C), even when excluding the relationships with other resilience factors (22.8-15.7\%). However, in the latter situation, the estimation of the difference was relatively unstable, resulting in a large quantile interval that contained zero $(p>0.05$; Figures $\mathbf{6 B}, \mathbf{D})$. The connection of MUL with the PPF facet $(7.7 \%$ vs. $6.4 \%, p>0.05)$ was of particular interest, since PPF was not directly related to overall SMA in the second GGM. Moreover, this connection appeared to be stronger than the edge between PPF and PAS, although not significantly $(p>0.05$; Supplementary Figures 9, 10). These exploratory findings suggest that, potentially, when aiming to improve the PPF facet of QoL, one should focus on enhancing multifunctionality of resources specifically, rather than PAS. Due to the considerably high total outstrength value of PPF on other facets of QoL (79.0\%, vs. $48.4 \%$ instrength, $p<0.05)$, this may also be an excellent strategy to indirectly enhance AUT (29.7\%), SOP (29.3\%), INT (14\%), DAD (4.3\%), and SAB (1.7\%), and thereby QoL as a whole. Several other positive (and some negative) relationships between the individual SMAs and QoL facets were observed as well, although most of them were considerably unstable (see Figures 5, 6 and Supplementary Figures 9, 10).

In sum, these exploratory analyses again highlight that the selection of the most appropriate resilience factor to manipulate depends on the QoL facet one aims to promote. Most interestingly, it seems that targeting the multifunctionality facet of the SMAS can potentially have the strongest effect on overall QoL. This facet was strongly related to the PFF activities facet of QoL, which in turn exerts a large influence on multiple other QoL facets. 
A

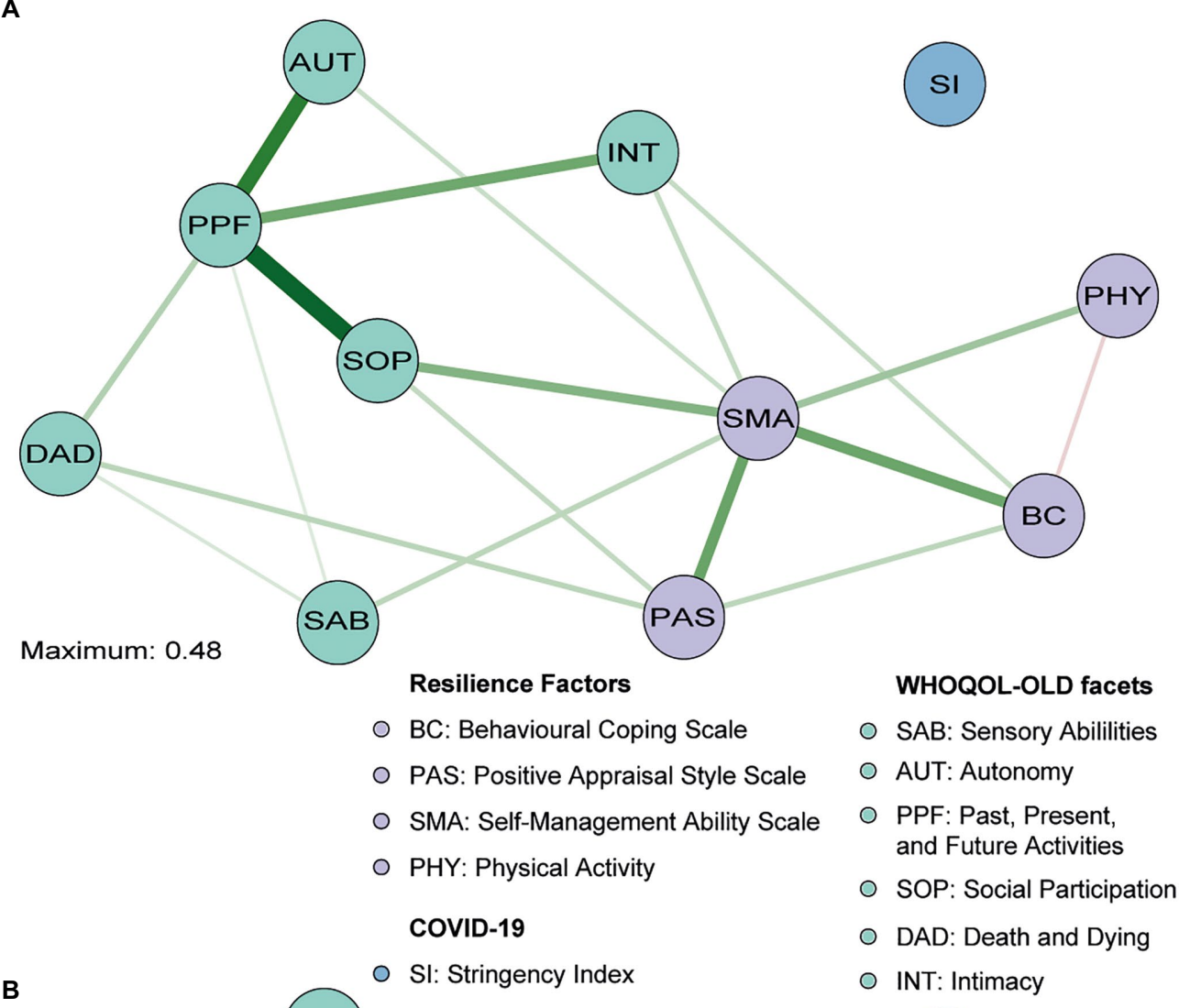

B

SI: Stringency Index

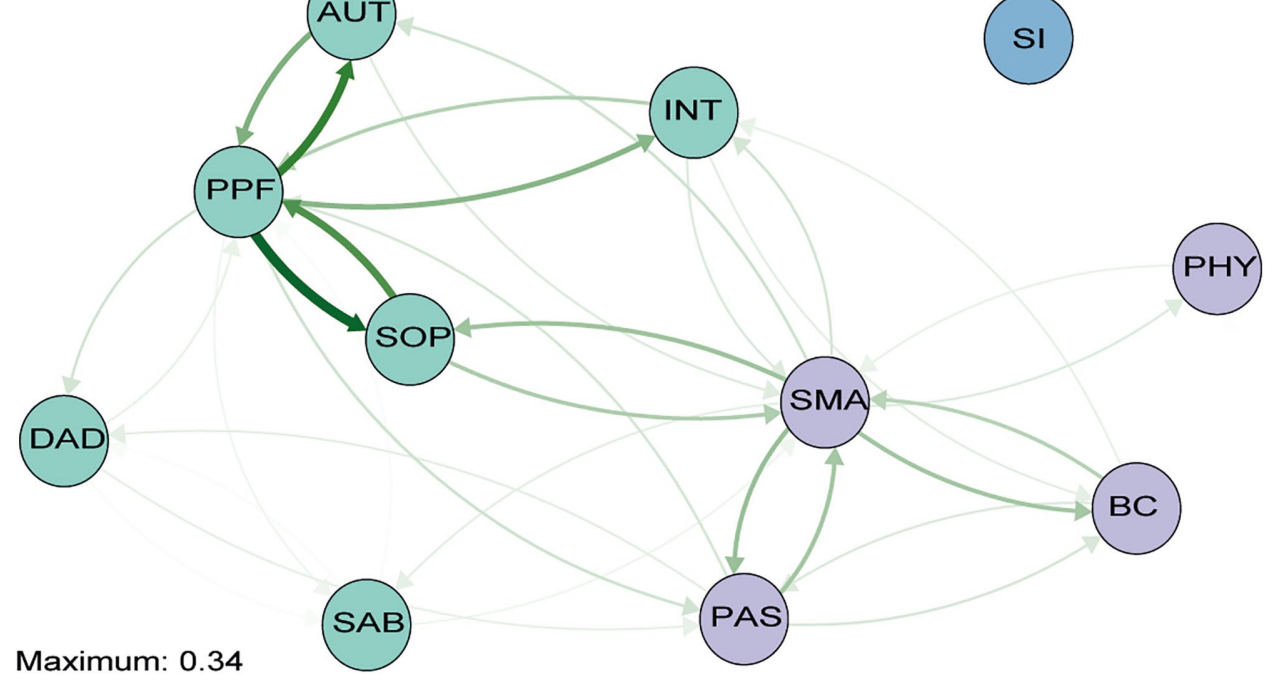

FIGURE 3 | Gaussian graphical model (GGM; A) and directed relative importance network (B) of individual facets of QoL (green), the resilience factors (purple), and the stringency index (blue). The maximum value represents the highest edge weights included in the network. Note that the direct relationship from positive appraisal (PAS) to past, present and future activities (PPF) is covered by the social participation (SOP) node and that PAS is thus not directly related to SOP (only indirectly via PPF).

\section{DISCUSSION}

The aging process is accompanied by challenges and transitions that can lead to significant changes in QoL if these are not adequately managed and regulated. The present study adopted a network approach to investigate the interplay between key resilience factors and QoL (as an outcome measure of resilience) in later life. Our expectation to find positive relationships between (facets of) QoL and our resilience factors of interest, either directly or indirectly, received strong support. We first 
A

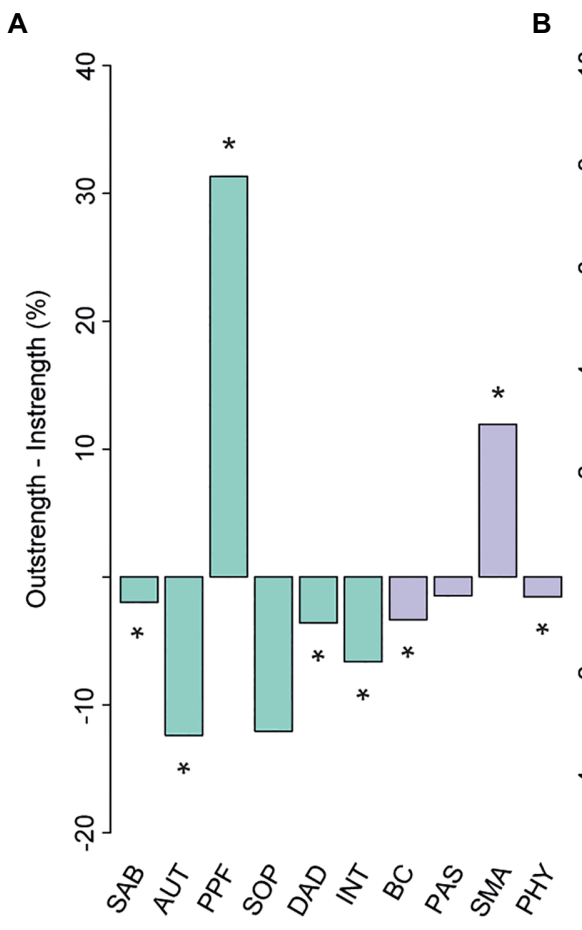

C

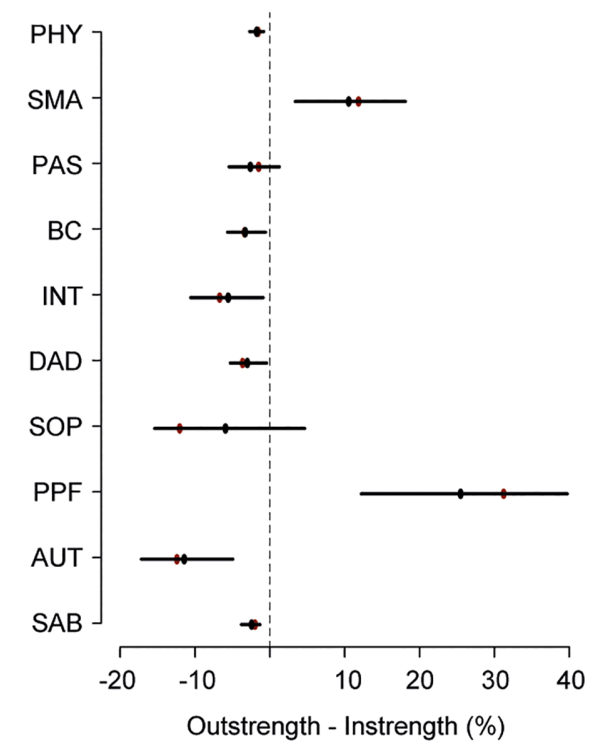

B

D
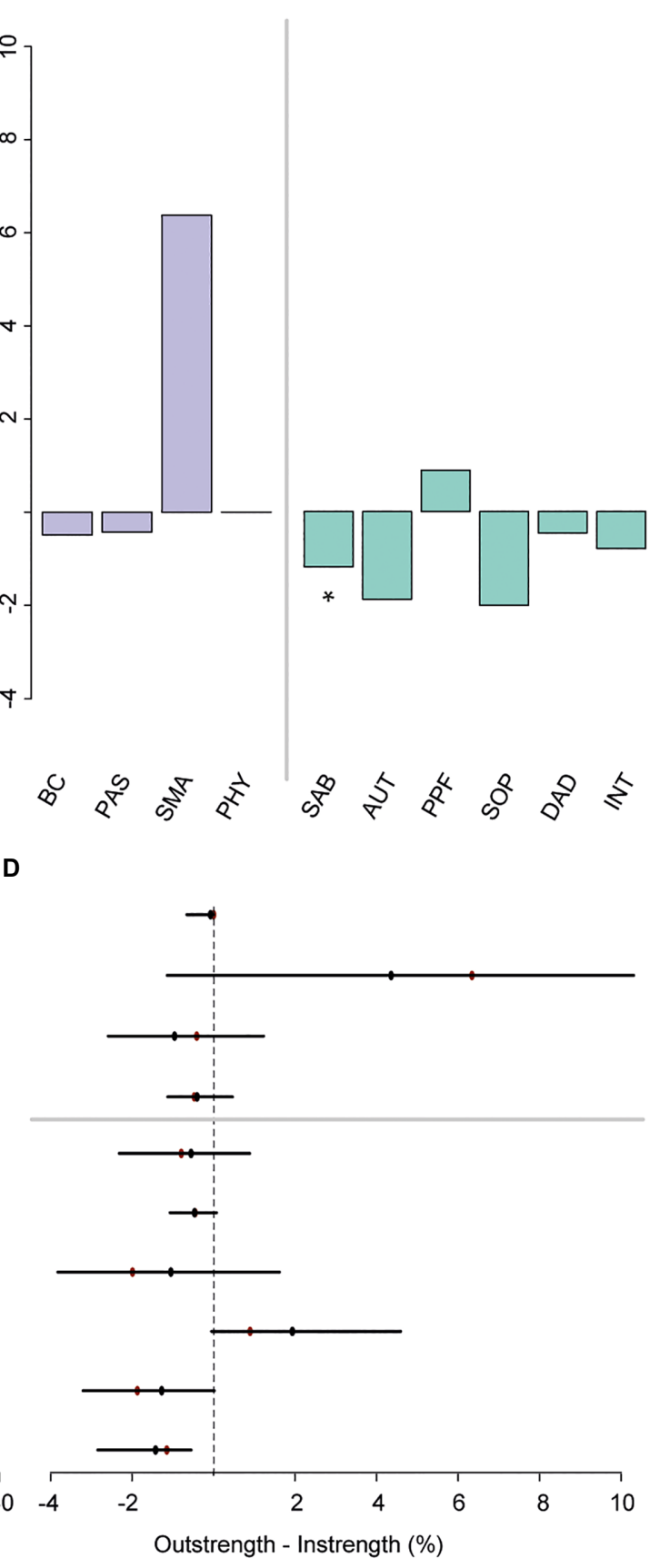

FIGURE 4 | The difference, including bootstrapped quantile intervals, between total outstrength and instrength of all the nodes in the secondary network (A,C), and the difference in total outstrength and instrength of the relationships between the resilience factors and QoL facets only (B, left; $\mathbf{D}$, top), and the relationships between the QoL facets and the resilience factors (B, right; $\mathbf{D}$, bottom). Colors of the bar charts correspond to the nodes in the network in Figure $\mathbf{3}$. In plots (C,D), the bootstrapped mean is depicted in black and the sample mean in red. ${ }^{*} p<0.05$, nodes with quantile intervals containing zero are deemed to have an insignificant instrength and outstrength difference.

present a brief summary of the findings, followed by more in-depth discussion of the observed relationships.

Most fundamentally, we provide evidence to suggest that individual differences in SMA and (to a slightly lesser extent) PAS play a crucial role in predicting QoL. The first set of analyses revealed that SMA was the most influential resilience factor, not only having direct relationships with QoL, but also serving as an important connecting link between QoL and other positive resources (e.g., behavioral coping, physical activity). Moreover, both SMA and PAS exhibited a high relative importance as predictor of QoL (i.e., outstrength on QoL), albeit that only for SMA this was higher than its instrength. 

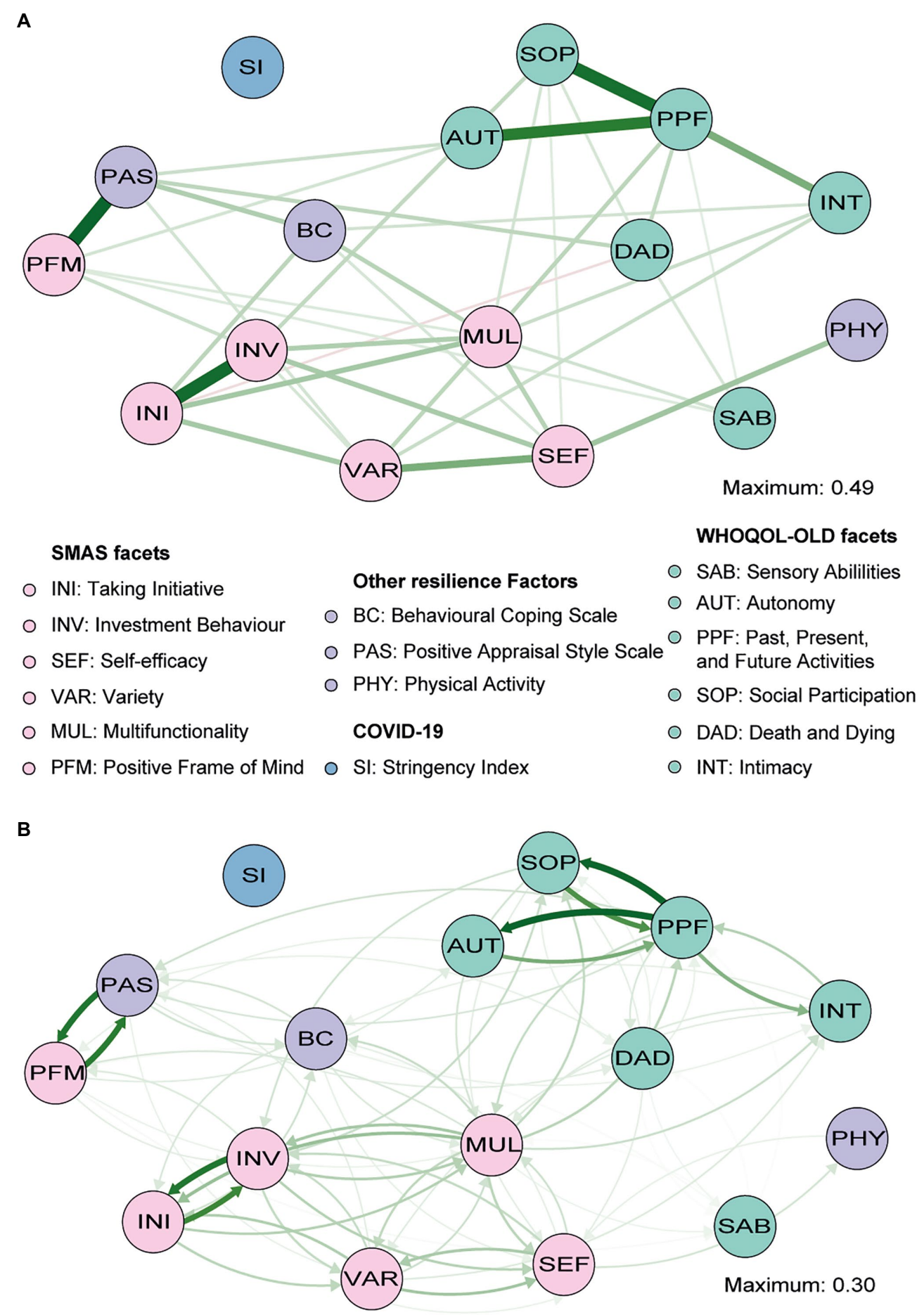

FIGURE 5 | Gaussian graphical model (GGM; A) and directed relative importance network (B) of individual facets of QoL (green), the facets of the SMAS (pink) and other resilience factors (purple), and the stringency index (blue). The maximum value represents the highest edge weights included in the network.

The relationship of SMA with QoL was driven by various underlying associations with multiple facets of QoL, including SAB, SOP, AUT, and INT. Moreover, while PAS appeared to influence overall QoL to a lesser extent than SMA, it was strongly connected to specific facets, namely DAD and
PPF. Therefore, manipulation of PAS may be an effective pathway for decreasing worries about DAD and improving satisfaction about achievements in life and at things to look forward to. However, exploratory analyses revealed that specifically targeting the multifunctionality (MUL) facet of 

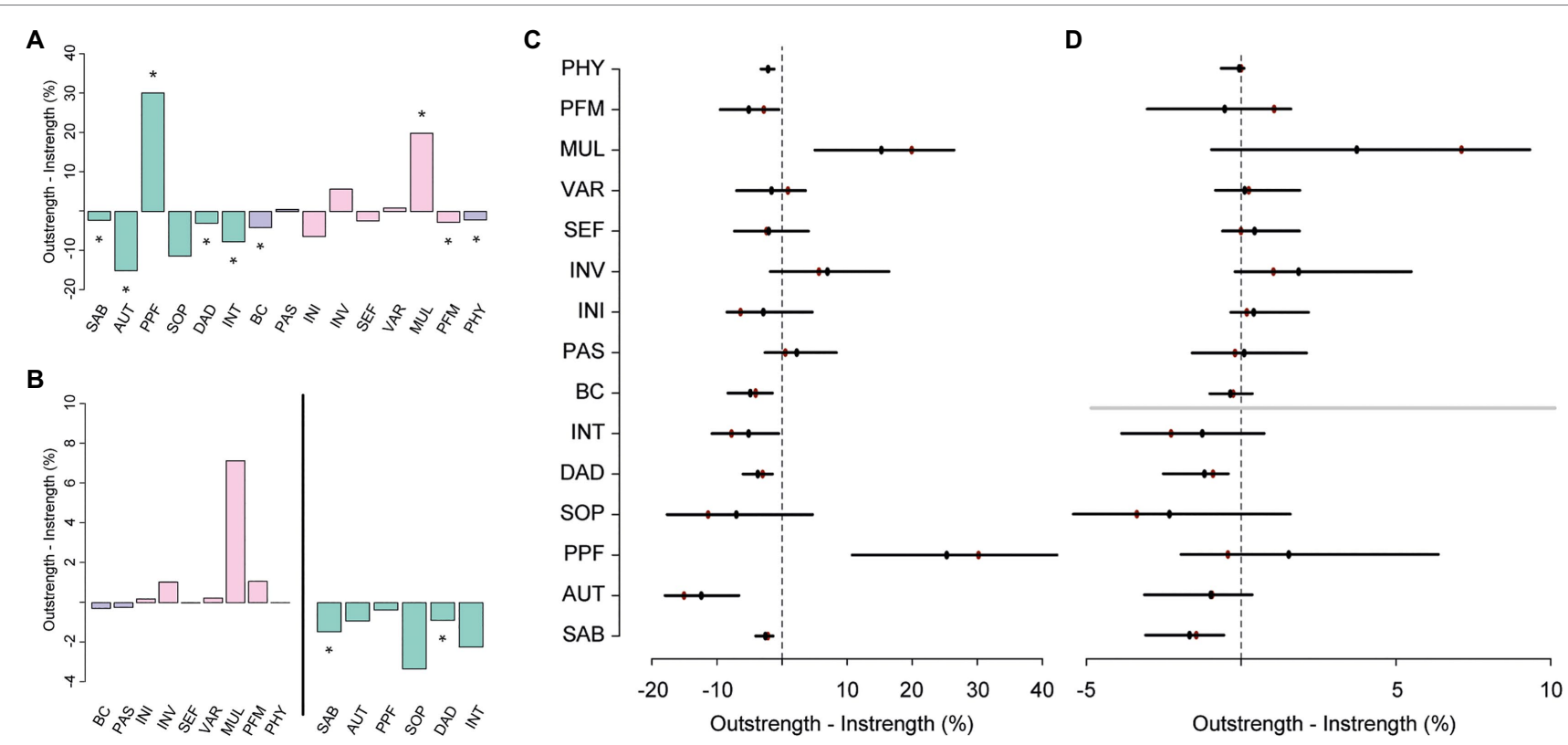

FIGURE 6 | The difference between overall outstrength and instrength of the nodes in the third network (A), and the difference in outstrength and instrength of the relationships between the resilience factors and QoL facets only (B, left), and the relationships between the QoL facets and the resilience factors (B, right). Colors correspond to the nodes in the network in Figure $\mathbf{5}$. In plots (C,D), the bootstrapped mean is depicted in black and the sample mean in red. ${ }^{*} p<0.05$, nodes with quantile intervals containing zero are deemed to have an insignificant instrength and outstrength difference.

the SMAS could also be a promising strategy to improve reports on PPF. Considering the large influence that PPF exerts on other QoL facets, MUL may be an excellent target for interventions or individuals' own efforts to promote overall QoL.

\section{Main (and Exploratory) Findings}

Our main finding that SMA is strongly associated with (multiple facets of) QoL (especially SOP) extends earlier demonstrations of positive associations between SMA and well-being and other health indicators (e.g., frailty, loneliness) at old age (Schuurmans et al., 2005; Cramm et al., 2012a,b, 2014; Nieboer et al., 2020). Moreover, our finding is in line with Cramm and Nieboer (2017) that successfully increased QoL by boosting SMA. The connections of SMA with other resilience factors, with SMA having a high relative importance as predictor of these factors, also suggest that a strong capacity to take care of one's life and external resources indirectly improves QoL by promoting other factors that can enhance QoL. Individuals with a strong SMA may have a richer repertoire of cognitive or behavioral coping abilities, but the effective implementation of SMAs may also provide opportunities or optimal circumstances to effectively employ specific coping strategies (e.g., many BC strategies require involvement of others; positively appraising a situation may be easier when one generally feels in control of their own life). As such, SMA may play an important role in the reinforcing spirals (or feedback loops) among the resilience factors. This is in line with the idea that resilience should be represented as a combination of protective factors that do not function in isolation, but are interconnected and (potentially) strengthen one another (e.g., Fritz et al., 2018, 2019; Scheffer et al., 2018; Brinkhof et al., 2021).

In addition to this main finding, the exploratory analyses on the individual SMAs revealed that MUL was relatively strongly connected to the PPF facet of QoL. This implies that the ability to achieve and maintain multifunctionality at old age contributes to the extent to which one is satisfied with PFF. Potentially, individuals with a strong MUL more frequently experience the synergetic effect created by mutually reinforcing activities or interactions, and as a consequence, they are more satisfied with achievements in life and things to look forward to. This is in accordance with the idea that the maintenance of social roles and activities that are meaningful to people enhances their feelings of well-being (Lemon et al., 1972; Winningham and Pike, 2007). Since PFF has a large outstrength on other facets of QoL, MUL may be a critical target for interventions aiming to improve overall QoL. Indeed, the chances of experiencing declines in multifunctional resources and activities increase with age (Steverink et al., 2005). This may be a consequence of the loss of a beloved person, but also fewer opportunities for multifunctionality (e.g., interaction with colleagues, physical exercise within a group). Hence, building interventions that help individuals to promote SMA, and multifunctionality in particular, thereby reducing potential declines in QoL (and well-being), is highly important.

One of the few existing interventions that has been developed for this purpose is the self-management of well-being (SMW) intervention, tested in different formats (individual, group, and self-help; Schuurmans, 2004; Frieswijk et al., 2006; Kremers et al., 2006; see Goedendorp and Steverink, 2017 for comparison). 
However, this intervention is high intensive, involving multiple (5-6) session (of 1-2.5h). To improve the accessibility for older adults, it may be useful to explore possibilities for low-intensity interventions that focus on the promotion of (simple) daily life adjustments in favor of multifunctionality specifically. For example, the strategic planning technique of implementation intentions could be used to link daily situations to a desired multifunctional activity (e.g., planning dinner with friends; participating in volunteer work; Gollwitzer, 1999). Such behavior change interventions may in turn promote socially rewarding and productive interactions among peers (Steverink et al., 2005).

Although to a lesser extent than SMA, PAS was also strongly and directly associated with QoL. This is in line with the notion that PAS is protective for mental health (Kalisch et al., 2015) and with earlier research showing that the use of cognitive (emotion regulation) coping can contribute to positive (mental) health outcomes at old age specifically (Garnefski et al., 2002; Kraaij et al., 2002; Garnefski and Kraaij, 2006b; Swift et al., 2008; Nowlan et al., 2015, 2016). Positive reappraisal most frequently emerged as adaptive strategy in these studies. It involves finding a positive meaning within a negative situation and has been shown to be effectively implemented by older adults (Shiota and Levenson, 2009; Hall et al., 2010; Nowlan et al., 2015). Hence, this strategy seems to be a good starting point for interventions intended to improve overall QoL (e.g., mindfulness; Garland et al., 2009), but possibly also to improve satisfaction with achievements in life and things to look forward to (PFF) and to reduce concerns, worries, and fears about $\mathrm{DAD}$, specifically. Indeed, previous studies have found positive associations with overall life satisfaction (Swift et al., 2008; Windsor, 2009), and the few existing reports on coping with death anxiety have claimed that strategies involving positive re-evaluation or re-organization of thinking should be a main component of treatment (Cicirelli, 2003; Purer and Walker, 2008). Since our research points toward a general benefit of a PAS, we suggest future studies to examine the intervening potential of this generic tendency as well.

Our results also suggest that PAS contributes more strongly to QoL than BC. BC was also positively related to QoL, but this was mediated by PAS (and SMA), which exhibited a larger relative importance as predictor of $\mathrm{BC}$ than vice versa. This is in line with earlier suggestions that a focus on changing one's emotional response, rather than managing the situation that elicited them, is more adaptive at old age, since many challenges and event in late life are negative and often unalterable (Folkman and Lazarus, 1980; Cheng, 2001; cf. Nowlan et al., 2015's perspective on positive reappraisal). Moreover, considering the fact that social networks tend to decline with age (Wrzus et al., 2013) and that many items of the BCS reflect socially supported coping (i.e., requiring the presence or active involvement of others; Nahlen Bose et al., 2015; Ingrand et al., 2018), BC may also be less favorable in later life. In line with this, the observed relationship between BC and the INT facet of QoL may be explained by the fact that the perceived level of intimacy is inherently associated with the number of people someone can call on in difficult situations. As such, BC strategies may only be successfully implemented by those already experiencing high levels of INT, rendering $\mathrm{BC}$ ineffective as strategy to improve levels of intimacy.
Accordingly, it has been suggested that aging is accompanied by a general shift from the use of $\mathrm{BC}$, centered on problems and seeking of assistance, to cognitive- and emotion-focused coping (Folkman et al., 1987; Blanchard-Fields and Irion, 1988; Blanchard-Fields et al., 1995; Watson and Blanchard-Fields, 1998; Garnefski et al., 2001; Chen et al., 2018; Ingrand et al., 2018). Still, the effectiveness of BC may ultimately also differ between aging individuals, with different sizes of social networks one can resort to, which should be addressed in future studies.

Our findings provide relatively strong support for our hypothesis that physical activity has a positive effect on QoL (e.g., Windle et al., 2010), either directly or by promoting other resilience factors (e.g., Ávila et al., 2018). That is, PHY was positively associated and linked to SMA, and therefore indirectly to QoL, but the relative importance as a predictor of SMA was low in comparison with its instrength (from SMA). This implies that SMA mediated the relationship between PHY and QoL, but that the contribution of SMA on PHY was larger. This is in line with the idea that strengthening resilience can improve the adherence to exercise behaviors (Resnick and Inguito, 2011). Interestingly, our exploratory analyses revealed that the relationship between PHY and SMA was driven by self-efficacy specifically, which conforms with earlier studies claiming that targeting self-efficacy is the most effective means to increase physical activity (e.g., van Stralen et al., 2009). Moreover, this finding is consistent with the growing number of studies reporting an indirect effect of PHY on QoL, through self-efficacy (Rejeski and Mihalko, 2001; Elavsky et al., 2005; McAuley et al., 2006; White et al., 2009; Phillips et al., 2013). Thus, enhancing the belief in one's personal competence to achieve goals in life seems highly beneficial to improve overall levels of QoL, as well as physical activity (see Williams and French, 2011 for examples on how to achieve this). The fact that PHY had no direct associations with QoL remains surprising. A possible explanation may be that the current measure of PHY was too general and did not pick up decisive differences among individuals. This has been shown to be a general pitfall of self-report measures of physical activity (Prince et al., 2008), which emphasizes that cautious interpretation is warranted.

The lack of an effect of the stringency of the COVID-19 policies on QoL may be explained by the fact that our study took place during the second wave of the pandemic. Previous studies that were conducted during the initial phase, or its aftermath (summer 2020), have reported either negative (O'Hara et al., 2020; Lee et al., 2021a; Voss et al., 2021), or positive effects on individuals' mental health (Kim and Jung, 2021; Lee et al., 2021b; Long et al., 2021). Hence, current findings may indicate that, over time, individuals became more resistant to fluctuations in policies and restrictions, possibly because they had become accustomed to the uncertainty and frequent policy changes. Another factor that could explain the discrepancy between the current and aforementioned reports is that we evaluated fluctuations within one country, while others looked at differences between countries (with more or less stringent measures). Interestingly, one previous finding that is in accordance with current observations is that of Ding et al. (2021), who found that after adjusting for personal risk factors, a more stringent governmental policy response was not associated with increased 
anxiety and depression symptoms during the lockdown. Clearly, taken together with the present findings, this suggests that it is important to evaluate multiple factors at once, yet the impact of the stringency index on (outcome based) resilience warrants further in-depth investigation.

\section{Limitations and Future Directions}

Since our analyses relied on cross-sectional data, we could not confirm causal relationships. Indeed, the networks provided clues as to how the resilience factors influenced one another and (facets of) QoL specifically, but intervention studies or other experimental manipulations are required to determine the true intervening potential of these factors. Therefore, the revealed patterns of associations, and hypotheses derived from these, may help guide future research (Epskamp et al., 2018b).

Moreover, the fact that our research took place during the COVID-19 pandemic may have influenced our findings in different ways. We show that fluctuations in COVID-19 policy strictness were too small to affect our measures, but additional research is needed to establish the full impact of the COVID-19 pandemic itself. That is, since our study only included data that were collected during COVID-19, it remains unclear whether a different pattern of associations would emerge if we would include data from after the brunt of the pandemic. Natural variation among individuals in physical activity may have been reduced, due to the restrictions adopted by the Dutch government that limited the opportunities and possibilities to engage in physical activity. Consequently, the (indirect) associations between physical activity and QoL could been affected. Similarly, the implementation of SMAs that focus on managing one's external resources in a world that is dominated by social restrictions may have been compromised, possibly resulting in different association patterns or at least a dampening of relationships. For example, there were severe restrictions on gathering which in turn limited opportunities to socialize (play sports, go out for dinner, etc.).

One other important constraint to be aware of is the fact that our sample is represented by a predominantly highly educated, native-born group of older adults. This may impact the generalizability of our results, as both education level and migration history have been shown to affect (outcome based) resilience among older adults in a number of previous studies (Lund et al., 2018; Klokgieters et al., 2020; Szabó et al., 2020). Hence, we encourage researchers to perform similar studies to reveal whether the pattern of associations of minority groups approximates the one observed in the current study. Such attempts may require careful considerations of reducing certain barriers (e.g., online, language) that may hold individuals back from participating, for instance by using translated, paper-and-pencil versions.

Further investigation will also be needed to establish whether age can moderate associations among the resilience factors and QoL (facets). Indeed, with advancing age, individuals face an increasing amount of challenges that have to be regulated and managed. This places a greater demand on their capacity for resilience, potentially putting the oldest individuals at higher risk to reach a certain tipping point beyond which they will experience considerable declines in QoL. On the other hand, research has also shown that old age is associated with more beneficial outcomes in the face of adversity (Wilson et al., 2020; Fuller and HusethZosel, 2021; Lind et al., 2021), in agreement with the notion that the accumulation of life events leads to an enlarged repertoire of knowledge and (coping) skills that can help the oldest individuals to deal with the challenges they are confronted with (Norris and Murrell, 1988; Seery et al., 2010; Crane et al., 2019). While the controversial question on the impact of age was beyond the scope of the current study, this important issue will need to be addressed in future work (within our own lab, for one).

Despite these limitations and constraints, the present study widens our understanding of the role of multiple resilience factors in predicting QoL as an outcome of resilience. Most fundamentally, our patterns of associations emphasize the complex and emergent nature of resilience (Brinkhof et al., 2021), showing that resilience in later life arises through interactions among several components, thereby impacting QoL jointly and possibly in a self-reinforcing way (Fritz et al., 2018, 2019; Scheffer et al., 2018). This highlights the importance of investigating the role of resilience factors simultaneously (in complex systems), rather than individually. Future work in our group will focus on improving our comprehension of these complex interactions by evaluating the role and contribution of a wide-ranging pallet of risk factors, in addition to multiple other resilience factors, as well. Adding those factors might change the structure of the network, resulting in a more thorough picture of the contribution of each node. Current relationships between resilience factors and QoL will likely still be obtained (e.g., PAS-QoL), only the pathways through which each factor influences QoL may be more branched than we can currently identify. Moreover, while the contribution of physical activity on the WHOQOL-OLD reports seems relatively low as compared to the other factors included in the current networks, its effects on more health-related QoL measures (e.g., SF-36) may be much stronger. By including both risk and protective factors, we can improve our understanding of the role of favorable and less favorable characteristics (e.g., low SES or availability of green space) or predispositions (e.g., history of depression, anxiety, and low self-esteem) within the overall network, and may, for instance, reveal interesting differences in association patterns between those that have experienced specific life events (such as the loss of a spouse) and those who are relatively unscathed.

\section{CONCLUDING REMARKS}

Summarizing, this study contributes to our understanding of the interplay between factors that underpin resilience in later life. We have provided evidence to suggest that SMA and, to a lesser extent, PAS are most crucially involved in the realization and maintenance of high levels of QoL, and building interventions targeting these factors therefore seems most promising when trying to improve QoL. Helping older adults to implement daily life adjustments that lead to socially rewarding and productive interactions with various benefits, may be an excellent starting point for interventions. However, the appropriate set of resilience factors to manipulate may ultimately depend on the facet of QoL that one intends to improve. These findings can aid future studies in determining specific strategies that can help older 
adults to gain control of their own lives, enabling them to maintain the functional ability and competence that is vital for well-being and QoL at old age.

\section{DATA AVAILABILITY STATEMENT}

The datasets presented in this article are not readily available because the datasets used and/or analyzed for the current study will only be made publicly available after completion of the overarching project, and will until that time only be available from the corresponding author on collaboration basis upon reasonable request. Requests to access the datasets should be directed to LB, l.p.brinkhof@uva.nl.

\section{ETHICS STATEMENT}

The studies involving human participants were reviewed and approved by the Local Ethics Committee of the University of Amsterdam (2020-DP-12556) and was conducted in accordance with relevant laws and institutional guidelines. The patients/ participants provided their written informed consent to participate in this study.

\section{AUTHOR CONTRIBUTIONS}

LB, KRR, JM, SW, and HK conceived the current study. LB, $\mathrm{KRR}$, and JM conceived the corresponding network analytical

\section{REFERENCES}

American Psychological Association (2020). "Resilience." in APA dictionary of psychology. Available at: https://dictionary.apa.org/resilience (Accessed February $8,2021)$.

Arroll, B., and Beaglehole, R. (1992). Does physical activity lower blood pressure: a critical review of the clinical trials. J. Clin. Epidemiol. 45, 439-447. doi: 10.1016/0895-4356(92)90093-3

Ávila, M. P. W., Corrêa, J. C., Lucchetti, A. L. G., and Lucchetti, G. (2018). The role of physical activity in the association between resilience and mental health in older adults. J. Aging Phys. Act. 26, 248-253. doi: 10.1123/japa.2016-0332

Bernstein, E. E., Heeren, A., and McNally, R. J. (2017). Unpacking rumination and executive control: a network perspective. Clin. Psychol. Sci. 5, 816-826. doi: $10.1177 / 2167702617702717$

Blanchard-Fields, F., and Irion, J. C. (1988). Coping strategies from the perspective of two developmental markers: age and social reasoning. J. Genet. Psychol. 149, 141-151. doi: 10.1080/00221325.1988.10532148

Blanchard-Fields, F., Jahnke, H. C., and Camp, C. (1995). Age differences in problem-solving style: the role of emotional salience. Psychol. Aging 10, 173-180. doi: 10.1037/0882-7974.10.2.173

Bollen, K. A., and Stine, R. A. (1992). Bootstrapping goodness-of-fit measures in structural equation models. Sociol. Methods Res. 21, 205-229. doi: 10.1177/0049124192021002004

Borsboom, D. (2017). A network theory of mental disorders. World Psychiatry 16, 5-13. doi: 10.1002/wps.20375

Bowling, A. (2007). Quality of Life in Older Age: What Older People Say. Dordrecht: Springer, 15-30.

Brinkhof, L. P., Ridderinkhof, K. R., de Wit, S., Krugers, H. J., and Murre, J. M. J. (2021). A network perspective on successful aging and resilience in later life: a protocol paper. PsyArXiv [Preprint]. doi: 10.31234/osf.io/bu7vq framework. LB implemented the study. LB and $\mathrm{KH}$ performed the statistical analysis. All authors contributed to the article and approved the submitted version.

\section{FUNDING}

This research is part of the "Active and Healthy Aging" project, which was funded by the Centre for Urban Mental Health, a Research Priority Area at the University of Amsterdam.

\section{PREPRINT}

The preprint of this manuscript can be found at https://psyarxiv. com/6vmz9/.

\section{ACKNOWLEDGMENTS}

We would like to thank our participants who took part in this study.

\section{SUPPLEMENTARY MATERIAL}

The Supplementary Material for this article can be found online at: https://www.frontiersin.org/articles/10.3389/fpsyg.2021.752564/ full\#supplementary-material

Carver, C. S. (1997). You want to measure coping but your protocol's too long: consider the brief COPE. Int. J. Behav. Med. 4, 92-100. doi: 10.1207/ s15327558ijbm0401_6

Chen, Y., Peng, Y., Xu, H., and O'Brien, W. H. (2018). Age differences in stress and coping: problem-focused strategies mediate the relationship between age and positive affect. Int. J. Aging Hum. Dev. 86, 347-363. doi: $10.1177 / 0091415017720890$

Cheng, C. (2001). Assessing coping flexibility in real-life and laboratory settings: a multimethod approach. J. Pers. Soc. Psychol. 80, 814-833. doi: 10.1037/0022-3514.80.5.814

Childs, E., and de Wit, H. (2014). Regular exercise is associated with emotional resilience to acute stress in healthy adults. Front. Physiol. 5:161. doi: 10.3389/ fphys.2014.00161

Chmitorz, A., Kunzler, A., Helmreich, I., Tüscher, O., Kalisch, R., Kubiak, T., et al. (2018). Intervention studies to foster resilience - a systematic review and proposal for a resilience framework in future intervention studies. Clin. Psychol. Rev. 59, 78-100. doi: 10.1016/j.cpr.2017.11.002

Cicirelli, V. G. (2003). Older adults' fear and acceptance of death: a transition model. Ageing Int. 28, 66-81. doi: 10.1007/s12126-003-1016-6

Clark, P. G., Burbank, P. M., Greene, G., Owens, N., and Riebe, D. (2011). "What do we know about resilience in older adults? An exploration of some facts, factors, and facets," in Resilience in Aging: Concepts, Research, and Outcomes. eds. B. Resnick, L. Gwyther and K. Roberto (New York: Springer), 51-66.

Cohen, J. (1977). Statistical Power Analysis for the Behavioural Sciences. New York: Academic Press.

Conn, V. S., Hafdahl, A. R., and Brown, L. M. (2009). Meta-analysis of qualityof-life outcomes from physical activity interventions. Nurs. Res. 58, 175-183. doi: 10.1097/NNR.0b013e318199b53a

Cramm, J. M., Hartgerink, J. M., de Vreede, P. L., Bakker, T. J., Steyerberg, E. W., Mackenbach, J. P., et al. (2012a). The relationship between older adults 
self-management abilities, well-being and depression. Eur. J. Ageing 9, 353-360. doi: 10.1007/s10433-012-0237-5

Cramm, J. M., and Nieboer, A. P. (2017). Self-management abilities and quality of life among frail community-dwelling individuals: the role of community nurses in the Netherlands. Health Soc. Care Commun. 25, 394-401. doi: $10.1111 /$ hsc. 12318

Cramm, J. M., Strating, M. M., de Vreede, P. L., Steverink, N., and Nieboer, A. P. (2012b). Validation of the self-management ability scale (SMAS) and development and validation of a shorter scale (SMAS-S) among older patients shortly after hospitalisation. Health Qual. Life Outcomes 10:9. doi: 10.1186/1477-7525-10-9

Cramm, J. M., Twisk, J., and Nieboer, A. P. (2014). Self-management abilities and frailty are important for healthy aging among community-dwelling older people: a cross-sectional study. BMC Geriatr. 14:28. doi: 10.1186/1471-2318-14-28

Crane, M. F., Searle, B. J., Kangas, M., and Nwiran, Y. (2019). How resilience is strengthened by exposure to stressors: the systematic self-reflection model of resilience strengthening. Anxiety Stress Coping 32, 1-17. doi: 10.1080/10615806.2018.1506640

Ding, K., Yang, J., Chin, M. K., Sullivan, L., Demirhan, G., Violant-Holz, V., et al. (2021). Article mental health among adults during the covid-19 pandemic lockdown: a cross-sectional multi-country comparison. Int. J. Environ. Res. Public Health 18, 1-16. doi: 10.3390/ijerph18052686

Efron, B. (1979). Bootstrap methods: another look at the jackknife. Ann. Stat. 7, $1-26$.

Ekerdt, D. J., Koss, C. S., Li, A., Münch, A., Lessenich, S., and Fung, H. H. (2017). Is longevity a value for older adults? J. Aging Stud. 43, 46-52. doi: 10.1016/j.jaging.2017.10.002

Elavsky, S., McAuley, E., Motl, R. W., Konopack, J. F., Marquez, D. X., Hu, L., et al. (2005). Physical activity enhances long-term quality of life in older adults: efficacy, esteem, and affective influences. Ann. Behav. Med. 30, 138-145. doi: 10.1207/s15324796abm3002_6

Endler, N. S., and Parker, J. D. A. (1990). Multidimensional assessment of coping: a critical evaluation. J. Pers. Soc. Psychol. 58, 844-854. doi: 10.1037/0022-3514.58.5.844

Epskamp, S., Borsboom, D., and Fried, E. I. (2018a). Estimating psychological networks and their accuracy: a tutorial paper. Behav. Res. Methods 50, 195-212. doi: 10.3758/s13428-017-0862-1

Epskamp, S., Cramer, A. O. J., Waldorp, L. J., Schmittmann, V. D., and Borsboom, D. (2012). Qgraph: network visualizations of relationships in psychometric data. J. Stat. Softw. 48, 1-18. doi: 10.18637/jss.v048.i04

Epskamp, S., Waldorp, L. J., Mõttus, R., and Borsboom, D. (2018b). The Gaussian graphical model in cross-sectional and time-series data. Multivar. Behav. Res. 53, 453-480. doi: 10.1080/00273171.2018.1454823

Figueira, H. A., Cader, S. A., Oliveira, R. J., Figueira, J. A., Figueira, O. A., Dantas, E. H. M., et al. (2012). Effects of a physical activity governmental health programme on the quality of life of elderly people. Scand. J. Public Health 40, 418-422. doi: 10.1177/1403494812453885

Folkman, S., and Lazarus, R. S. (1980). An analysis of coping in a middle-aged community sample. J. Health Soc. Behav. 21, 219-239. doi: 10.2307/2136617

Folkman, S., Lazarus, R. S., Pimley, S., and Novacek, J. (1987). Age differences in stress and coping processes. Psychol. Aging 2, 171-184. doi: 10.1037/0882-7974.2.2.171

Fontes, A. P., and Neri, A. L. (2015). Resilience in aging: literature review. Cien. Saude Colet. 20, 1475-1495. doi: 10.1590/1413-81232015205.00502014

Foygel, R., and Drton, M. (2010). "Extended Bayesian information criteria for Gaussian graphical models.” in arXiv preprint. Available at: http://arxiv.org/ abs/1011.6640

Fried, E. I., Bockting, C., Arjadi, R., Borsboom, D., Amshoff, M., Cramer, A. O. J., et al. (2015). From loss to loneliness: the relationship between bereavement and depressive symptoms. J. Abnorm. Psychol. 124, 256-265. doi: 10.1037/ abn 0000028

Frieswijk, N., Steverink, N., Buunk, B. P., and Slaets, J. P. J. (2006). The effectiveness of a bibliotherapy in increasing the self-management ability of slightly to moderately frail older people. Patient Educ. Couns. 61, 219-227. doi: $10.1016 /$ j.pec.2005.03.011

Fritz, J., Fried, E. I., Goodyer, I. M., Wilkinson, P. O., and van Harmelen, A. L. (2018). A network model of resilience factors for adolescents with and without exposure to childhood adversity. Sci. Rep. 8:15774. doi: 10.1038/ s41598-018-34130-2
Fritz, J., Stochl, J., Fried, E. I., Goodyer, I. M., Van Borkulo, C. D., Wilkinson, P. O., et al. (2019). Unravelling the complex nature of resilience factors and their changes between early and later adolescence. BMC Med. 17:203. doi: 10.1186/ s12916-019-1430-6

Fruchterman, T. M. J., and Reingold, E. M. (1991). Graph drawing by forcedirected placement. Softw. Pract. Exp. 21, 1129-1164. doi: 10.1002/spe.4380211102

Fry, P. S., and Keyes, C. L. M. (eds.) (2010). "New frontiers in resilient aging: life-strengths and well-being in late life," in New Frontiers in Resilient Aging: Life-Strengths and Well-Being in Late Life. Cambridge University Press.

Fuller, H. R., and Huseth-Zosel, A. (2021). Lessons in resilience: initial coping among older adults during the COVID-19 pandemic. Gerontologist 61, 114-125. doi: 10.1093/geront/gnaa170

Gamrowska, A., and Steuden, S. (2014). Coping with the events of daily life and quality of life of the socially active elderly. Health Psychol. Rep. 2, 123-131. doi: 10.5114/hpr.2014.43919

Garland, E., Gaylord, S., and Park, J. (2009). The role of mindfulness in positive reappraisal. Explore 5, 37-44. doi: 10.1016/j.explore.2008.10.001

Garnefski, N., and Kraaij, V. (2006a). Cognitive emotion regulation questionnaire - development of a short 18-item version (CERQ-short). Personal. Individ. Differ. 41, 1045-1053. doi: 10.1016/j.paid.2006.04.010

Garnefski, N., and Kraaij, V. (2006b). Relationships between cognitive emotion regulation strategies and depressive symptoms: a comparative study of five specific samples. Personal. Individ. Differ. 40, 1659-1669. doi: 10.1016/j. paid.2005.12.009

Garnefski, N., Kraaij, V., and Spinhoven, P. (2001). Negative life events, cognitive emotion regulation and emotional problems. Personal. Individ. Differ. 30, 1311-1327. doi: 10.1016/S0191-8869(00)00113-6

Garnefski, N., Legerstee, J., Kraaij, V., Van Den Kommer, T., and Teerds, J. (2002). Cognitive coping strategies and symptoms of depression and anxiety: a comparison between adolescents and adults. J. Adolesc. 25, 603-611. doi: 10.1006/jado.2002.0507

Gerino, E., Rollè, L., Sechi, C., and Brustia, P. (2017). Loneliness, resilience, mental health, and quality of life in old age: a structural equation model. Front. Psychol. 8:2003. doi: 10.3389/fpsyg.2017.02003

Glisky, E. L. (2007). "Changes in cognitive function in human aging," in Brain Aging. ed. D. R. Riddle (Boca Raton: CRC Press), 3-20.

Gobbens, R. J. J., and van Assen, M. A. L. M. (2016). Psychometric properties of the Dutch WHOQOL-OLD. Health Qual. Life Outcomes 14:103. doi: 10.1186/s12955-016-0508-5

Goedendorp, M. M., and Steverink, N. (2017). Interventions based on selfmanagement of well-being theory: pooling data to demonstrate mediation and ceiling effects, and to compare formats. Aging Ment. Health 21, 947-953. doi: $10.1080 / 13607863.2016 .1182967$

Gollwitzer, P. M. (1999). Implementation intentions: strong effects of simple plans. Am. Psychol. 54, 493-503. doi: 10.1037/0003-066X.54.7.493

Goodwin, R. D. (2003). Association between physical activity and mental disorders among adults in the United States. Prev. Med. 36, 698-703. doi: 10.1016/S0091-7435(03)00042-2

Greve, W., and Staudinger, U. M. (2006). "Resilience in later adulthood and old age: resources and potentials for successful aging," in Developmental Psychopathology: Second Edition. Vol. 3. eds. D. Chichetti and D. J. Cohen (John Wiley \& Sons), 796-840.

Grömping, U. (2006). Relative importance for linear regression in R: the package relaimpo. J. Stat. Softw. 17, 1-27. doi: 10.18637/jss.v017.i01

Hall, N. C., Chipperfield, J. G., Heckhausen, J., and Perry, R. P. (2010). Control striving in older adults with serious health problems: a 9-year longitudinal study of survival, health, and well-being. Psychol. Aging 25, 432-445. doi: $10.1037 / \mathrm{a} 0019278$

Hanley, A. W., and Garland, E. L. (2014). Dispositional mindfulness co-varies with self-reported positive reappraisal. Personal. Individ. Differ. 66, 146-152. doi: $10.1016 /$ j.paid.2014.03.014

Harris, P. B. (2008). Another wrinkle in the debate about successful aging: the undervalued concept of resilience and the lived experience of dementia. Int. J. Aging Hum. Dev. 67, 43-61. doi: 10.2190/AG.67.1.c

Hastie, T., Tibshirani, R., and Wainwright, M. (2015). Statistical Learning With Sparsity: The Lasso and Generalizations. Boca Raton: CRC Press.

Hayman, K. J., Kerse, N., and Consedine, N. S. (2017). Resilience in context: the special case of advanced age. Aging Ment. Health 21, 577-585. doi: $10.1080 / 13607863.2016 .1196336$ 
Heeren, A., and McNally, R. J. (2016). An integrative network approach to social anxiety disorder: the complex dynamic interplay among attentional bias for threat, attentional control, and symptoms. J. Anxiety Disord. 42, 95-104. doi: 10.1016/j.janxdis.2016.06.009

Hildon, Z., Montgomery, S. M., Blane, D., Wiggins, R. D., and Netuveli, G. (2010). Examining resilience of quality of life in the face of health-related and psychosocial adversity at older ages: what is 'right' about the way we age? Gerontologist 50, 36-47. doi: 10.1093/geront/gnp067

Hildon, Z., Smith, G., Netuveli, G., and Blane, D. (2008). Understanding adversity and resilience at older ages. Sociol. Health Illn. 30, 726-740. doi: 10.1111/j. 1467-9566.2008.01087.x

Ho, F. K. W., Louie, L. H. T., Chow, C. B., Wong, W. H. S., and Ip, P. (2015). Physical activity improves mental health through resilience in Hong Kong Chinese adolescents. BMC Pediatr. 15:48. doi: 10.1186/ s12887-015-0365-0

Hoorelbeke, K., Marchetti, I., De Schryver, M., and Koster, E. H. W. (2016). The interplay between cognitive risk and resilience factors in remitted depression: a network analysis. J. Affect. Disord. 195, 96-104. doi: 10.1016/j. jad.2016.02.001

Ingrand, I., Paccalin, M., Liuu, E., Gil, R., and Ingrand, P. (2018). Positive perception of aging is a key predictor of quality-of-life in aging people. PLoS One 13:e0204044. doi: 10.1371/journal.pone.0204044

Isvoranu, A.-M., and Epskamp, S. (2021). Continuous and ordered categorical data in network psychometrics: which estimation method to choose? PsyArXiv [Preprint]. 1-16. doi: 10.31234/OSF.IO/MBYCN

Janssen, I., Heymsfield, S. B., and Ross, R. (2002). Low relative skeletal muscle mass (sarcopenia) in older persons is associated with functional impairment and physical disability. J. Am. Geriatr. Soc. 50, 889-896. doi: 10.1046/j.1532-5415.2002.50216.x

Jaul, E., and Barron, J. (2017). Age-related diseases and clinical and public health implications for the 85 years old and over population. Front. Public Health 5:335. doi: 10.3389/fpubh.2017.00335

Kalisch, R., Cramer, A. O. J., Binder, H., Fritz, J., Leertouwer, I., Lunansky, G., et al. (2019). Deconstructing and reconstructing resilience: a dynamic network approach. Perspect. Psychol. Sci. 14, 765-777. doi: 10.1177/1745691619855637

Kalisch, R., Müller, M. B., and Tüscher, O. (2015). A conceptual framework for the neurobiological study of resilience. Behav. Brain Sci. 38:e92. doi: 10.1017/S0140525X1400082X

Kalisch, R., Veer, I., Yuen, K. S., Hendler, T., Myin-Germeys, I., Walter, H., et al. (2020). DynaCORE-C: The DynaMORE cross-sectional survey study on psychological resilience to the mental health consequences of the Corona crisis. Available at: https://osf.io/5xq9p (Accessed May 5, 2020).

Kim, H. H. S., and Jung, J. H. (2021). Social isolation and psychological distress during the COVID-19 pandemic: a cross-national analysis. Gerontologist 61, 103-113. doi: 10.1093/geront/gnaa168

Klokgieters, S. S., Van Tilburg, T. G., Deeg, D. J. H., and Huisman, M. (2020). The linkage between aging, migration, and resilience: resilience in the life of older Turkish and Moroccan immigrants. J. Gerontol. B Psychol. Sci. Soc. Sci. 75, 1113-1123. doi: 10.1093/geronb/gbz024

Kraaij, V., Pruymboom, E., and Garnefski, N. (2002). Cognitive coping and depressive symptoms in the elderly: a longitudinal study. Aging Ment. Health 6, 275-281. doi: 10.1080/13607860220142387

Kremers, I. P., Steverink, N., Albersnagel, F. A., and Slaets, J. P. J. (2006). Improved self-management ability and well-being in older women after a short group intervention. Aging Ment. Health 10, 476-484. doi: $10.1080 / 13607860600841206$

LASA Team (2020). Physical activity - Longitudinal Aging Study Amsterdam. Available at: https://lasa-vu.nl/topics/physical-activity/ (Accessed September 14, 2021)

Lazarus, R. S., and Folkman, S. (1984). Stress, Appraisal, and Coping. New York: Springer Publishing Company.

Lee, J. H., Lee, H., Kim, J. E., Moon, S. J., and Nam, E. W. (2021a). Analysis of personal and national factors that influence depression in individuals during the COVID-19 pandemic: a web-based cross-sectional survey. Glob. Health 17:3. doi: 10.1186/s12992-020-00650-8

Lee, Y., Lui, L. M. W., Chen-Li, D., Liao, Y., Mansur, R. B., Brietzke, E., et al. (2021b). Government response moderates the mental health impact of COVID-19: a systematic review and meta-analysis of depression outcomes across countries. J. Affect. Disord. 290, 364-377. doi: 10.1016/j.jad.2021.04.050
Lemon, B. W., Bengtson, V. L., and Peterson, J. A. (1972). An exploration of the activity theory of aging: activity types and life satisfaction among in-movers to a retirement community. J. Gerontol. 27, 511-523. doi: 10.1093/geronj/27.4.511

León-Navarrete, M. M., Flores-Villavicencio, M. E., Mendoza-Ruvalcaba, N., Colunga-Rodríguez, C., Salazar-Garza, M. L., Sarabia-López, L. E., et al. (2017). Coping strategies and quality of life in elderly population. Open J. Soc. Sci. 05, 207-216. doi: 10.4236/jss.2017.510017

Leys, C., Ley, C., Klein, O., Bernard, P., and Licata, L. (2013). Detecting outliers: do not use standard deviation around the mean, use absolute deviation around the median. J. Exp. Soc. Psychol. 49, 764-766. doi: 10.1016/j.jesp.2013.03.013

Lind, M., Bluck, S., and McAdams, D. P. (2021). More vulnerable? The life story approach highlights older people's potential for strength during the pandemic. J. Gerontol. B Psychol. Sci. Soc. Sci. 76, E45-E48. doi: 10.1093/ geronb/gbaal05

Long, D., Haagsma, J. A., Janssen, M. F., Yfantopoulos, J. N., Lubetkin, E. I., and Bonsel, G. J. (2021). Health-related quality of life and mental well-being of healthy and diseased persons in 8 countries: does stringency of government response against early COVID-19 matter? SSM Popul. Health 15:100913. doi: 10.1016/j.ssmph.2021.100913

Lund, C., Brooke-Sumner, C., Baingana, F., Baron, E. C., Breuer, E., Chandra, P., et al. (2018). Social determinants of mental disorders and the sustainable development goals: a systematic review of reviews. Lancet Psychiatry 5, 357-369. doi: 10.1016/S2215-0366(18)30060-9

MacLeod, S., Musich, S., Hawkins, K., Alsgaard, K., and Wicker, E. R. (2016). The impact of resilience among older adults. Geriatr. Nurs. 37, 266-272. doi: 10.1016/j.gerinurse.2016.02.014

McAuley, E., Konopack, J. F., Motl, R. W., Morris, K. S., Doerksen, S. E., and Rosengren, K. R. (2006). Physical activity and quality of life in older adults: influence of health status and self-efficacy. Ann. Behav. Med. 31, 99-103. doi: 10.1207/s15324796abm3101_14

McNally, R. J., Robinaugh, D. J., Wu, G. W. Y., Wang, L., Deserno, M. K., and Borsboom, D. (2015). Mental disorders as causal systems: a network approach to posttraumatic stress disorder. Clin. Psychol. Sci. 3, 836-849. doi: $10.1177 / 2167702614553230$

Mehta, M., Whyte, E., Lenze, E., Hardy, S., Roumani, Y., Subashan, P., et al. (2008). Depressive symptoms in late life: associations with apathy, resilience and disability vary between young-old and old-old. Int. J. Geriatr. Psychiatry 23, 238-243. doi: 10.1002/gps.1868

Nahlen Bose, C., Bjorling, G., Elfstrom, M. L., Persson, H., and Saboonchi, F. (2015). Assessment of coping strategies and their associations with health related quality of life in patients with chronic heart failure: the brief COPE restructured. Cardiol. Res. 6, 239-248. doi: 10.14740/cr385w

Netuveli, G., and Blane, D. (2008). Quality of life in older ages. Br. Med. Bull. 85, 113-126. doi: 10.1093/bmb/ldn003

Netuveli, G., Wiggins, R. D., Montgomery, S. M., Hildon, Z., and Blane, D. (2008). Mental health and resilience at older ages: bouncing back after adversity in the British household panel survey. J. Epidemiol. Community Health 62, 987-991. doi: 10.1136/jech.2007.069138

Newman, A. B., Haggerty, C. L., Goodpaster, B., Harris, T., Kritchevsky, S., Nevitt, M., et al. (2003). Strength and muscle quality in a well-functioning cohort of older adults: the health, aging and body composition study. J. Am. Geriatr. Soc. 51, 323-330. doi: 10.1046/j.1532-5415.2003.51105.x

Nieboer, A. P., Hajema, K., and Cramm, J. M. (2020). Relationships of selfmanagement abilities to loneliness among older people: a cross-sectional study. BMC Geriatr. 20:184. doi: 10.1186/s12877-020-01584-x

Nieboer, A., and Lindenberg, S. (2002). "Substitution, buffers and subjective well-being: a hierarchical approach," in The Universality of Subjective WellBeing Indicators: A Multidisciplinary and Multi-National Perspective. Social Indicators Book Series 16. eds. E. Gullone and R. A. Cummins (Dordrecht, NL: Kluwer Academic Publishers), 175-189.

Norris, F. H., and Murrell, S. A. (1988). Prior experience as a moderator of disaster impact on anxiety symptoms in older adults. Am. J. Community Psychol. 16, 665-683. doi: 10.1007/BF00930020

Nowlan, J. S., Wuthrich, V. M., and Rapee, R. M. (2015). Positive reappraisal in older adults: a systematic literature review. Aging Ment. Health 19, 475-484. doi: 10.1080/13607863.2014.954528

Nowlan, J. S., Wuthrich, V. M., and Rapee, R. M. (2016). The impact of positive reappraisal on positive (and negative) emotion among older adults. Int. Psychogeriatr. 28, 681-693. doi: 10.1017/S1041610215002057 
O'Hara, L., Abdul Rahim, H., and Shi, Z. (2020). Gender and trust in government modify: the association between mental health and stringency of public health measures to reduce COVID-19. MedRxiv [Preprint]. 305. doi: 10.29117/ quarfe.2020.0282

Ohrnberger, J., Fichera, E., and Sutton, M. (2017a). The dynamics of physical and mental health in the older population. J. Econ. Ageing 9, 52-62. doi: 10.1016/j.jeoa.2016.07.002

Ohrnberger, J., Fichera, E., and Sutton, M. (2017b). The relationship between physical and mental health: a mediation analysis. Soc. Sci. Med. 195, 42-49. doi: 10.1016/j.socscimed.2017.11.008

Phillips, S. M., Wójcicki, T. R., and McAuley, E. (2013). Physical activity and quality of life in older adults: an 18-month panel analysis. Qual. Life Res. 22, 1647-1654. doi: 10.1007/s11136-012-0319-z

Power, M., Quinn, K., and Schmidt, S. (2005). Development of the WHOQOLOLD module. Spring 14, 2197-2214. doi: 10.1007/s11136-005-7380-9

Prince, S. A., Adamo, K. B., Hamel, M. E., Hardt, J., Connor Gorber, S., and Tremblay, M. (2008). A comparison of direct versus self-report measures for assessing physical activity in adults: a systematic review. Int. J. Behav. Nutr. Phys. Act. 5:56. doi: 10.1186/1479-5868-5-56

Purer, P., and Walker, J. R. (2008). Death anxiety: a cognitive-behavioral approach. J. Cogn. Psychother. 22, 167-182. doi: 10.1891/0889-8391.22.2.167

Raut, N., Shanker, S., Singh, S., Subramanyam, A., Kamath, R., and Pinto, C. (2014). Study of loneliness, depression and coping mechanisms in elderly. J. Geriatr. Ment. Health 1:20. doi: 10.4103/2348-9995.141920

Rejeski, W. J., and Mihalko, S. L. (2001). Physical activity and quality of life in older adults. J Gerontol. A Biol. Sci. Med. Sci. 56(Suppl. 2), 23-35. doi: 10.1093/gerona/56.suppl_2.23

Resnick, B. A., and Inguito, P. L. (2011). The Resilience Scale: psychometric properties and clinical applicability in older adults. Arch. Psychiatr. Nurs. 25, 11-20. doi: 10.1016/j.apnu.2010.05.001

Rey, L., and Extremera, N. (2016). Forgiveness and health-related quality of life in older people: adaptive cognitive emotion regulation strategies as mediators. J. Health Psychol. 21, 2944-2954. doi: 10.1177/1359105315589393

Robinaugh, D. J., LeBlanc, N. J., Vuletich, H. A., and McNally, R. J. (2014). Network analysis of persistent complex bereavement disorder in conjugally bereaved adults. J. Abnorm. Psychol. 123, 510-522. doi: 10.1037/abn0000002

Ryff, C. D., Love, G. D., Essex, M. J., and Singer, B. (1998). "Resilience in adulthood and later life," in Handbook of Aging and Mental Health. ed. J. Lomranz (New York: Springer US), 69-96.

Satorres, E., Viguer, P., Fortuna, F. B., and Meléndez, J. C. (2018). Effectiveness of instrumental reminiscence intervention on improving coping in healthy older adults. Stress. Health 34, 227-234. doi: 10.1002/smi.2776

Scheffer, M., Elizabeth Bolhuis, J., Borsboom, D., Buchman, T. G., Gijzel, S. M. W., Goulson, D., et al. (2018). Quantifying resilience of humans and other animals. Proc. Natl. Acad. Sci. U. S. A. 115, 11883-11890. doi: 10.1073/ pnas. 1810630115

Schuurmans, H. (2004). Promoting well-being in frail elderly people: Theory and intervention [dissertation]. University of Groningen. Available at: https:// research.rug.nl/en/publications/promoting-well-being-in-frail-elderly-peopletheory-and-intervent

Schuurmans, H., Steverink, N., Frieswijk, N., Buunk, B. P., Slaets, J. P. J., and Lindenberg, S. (2005). How to measure self-management abilities in older people by self-report. The development of the SMAS-30. Qual. Life Res. 14, 2215-2228. doi: 10.1007/s11136-005-8166-9

Schuurmans, H., Steverink, N., Lindenberg, S., Frieswijk, N., and Slaets, J. P. (2004). Old or frail: what tells us more? J. Gerontol. Ser. A Biol. Med. Sci. 59, M962-M965. doi: 10.1093/gerona/59.9.M962

Seery, M. D., Holman, E. A., and Silver, R. C. (2010). Whatever does not kill us: cumulative lifetime adversity, vulnerability, and resilience. J. Pers. Soc. Psychol. 99, 1025-1041. doi: 10.1037/a0021344

Shiota, M. N., and Levenson, R. W. (2009). Effects of aging on experimentally instructed detached reappraisal, positive reappraisal, and emotional behavior suppression. Psychol. Aging 24, 890-900. doi: 10.1037/a0017896

Stephens, C., Breheny, M., and Mansvelt, J. (2015). Healthy ageing from the perspective of older people: a capability approach to resilience. Psychol. Health 30, 715-731. doi: 10.1080/08870446.2014.904862

Steverink, N., Lindenberg, S., and Slaets, J. P. J. (2005). How to understand and improve older people's self-management of wellbeing. Eur. J. Ageing 2, 235-244. doi: 10.1007/s10433-005-0012-y
Swift, A. U. A. A., Bailis, D. S., Chipperfield, J. G., Ruthig, J. C., and Newall, N. E. (2008). Gender differences in the adaptive influence of folk beliefs: a longitudinal study of life satisfaction in aging. Can. J. Behav. Sci. 40, 104-112. doi: 10.1037/0008-400X.40.2.104

Szabó, Á., Klokgieters, S. S., Kok, A. A. L., Van Tilburg, T. G., and Huisman, M. (2020). Psychological resilience in the context of disability: a study with Turkish and Moroccan young-old immigrants living in the Netherlands. Gerontologist 60, 259-269. doi: 10.1093/geront/gnz129

Taylor, D. (2014). Physical activity is medicine for older adults. Postgrad. Med. J. 90, 26-32. doi: 10.1136/postgradmedj-2012-131366

Upton, D., and Upton, P. (eds.) (2015). "Quality of life and well-being," in Psychology of Wounds and Wound Care in Clinical Practice (Cham: Springer International Publishing), 85-111.

Valtorta, N. K., Kanaan, M., Gilbody, S., Ronzi, S., and Hanratty, B. (2016). Loneliness and social isolation as risk factors for coronary heart disease and stroke: systematic review and meta-analysis of longitudinal observational studies. Heart 102, 1009-1016. doi: 10.1136/heartjnl-2015-308790

van Stralen, M. M., de Vries, H., Mudde, A. N., Bolman, C., and Lechner, L. (2009). Determinants of initiation and maintenance of physical activity among older adults: a literature review. Health Psychol. Rev. 3, 147-207. doi: 10.1080/17437190903229462

Vasconcelos, R., Souza, D. S., Carneiro, V., and Alves, D. (2016). Strength and ability to implement the activities of daily living in elderly resident in rural areas. Colomb. Med. 47, 167-171.

Veer, I., Riepenhausen, A., Zerban, M., Wackerhagen, C., Puhlmann, L., Engen, H., et al. (2020). Psycho-social factors associated with mental resilience in the Corona lockdown. PsyArXiv [Preprint]. doi: 10.31234/osf.io/4z62t

Vella, C., Kravitz, L., and Janot, J. (2001). A review of the impact of exercise on cholesterol levels. IDEA Health Fitness Source 19, 48-54.

Verhage, F. (1964). Intelligentie en leeftijd: Onderzoek bij Nederlanders van twaalf tot zevenenzeventig jaar [Intelligence and Age: Investigations on Dutch Persons from Twelve to Seventy-Seven Years]. Koninklijke Van Gorcum, Assen. Available at: https://research.rug.nl/en/publications/intelligentie-en-leeftijd-bijvolwassenen-en-bejaarden

Voss, G., Paiva, A. F., and Delerue Matos, A. (2021). A study of the association between the stringency of covid-19 government measures and depression in older adults across Europe and Israel. Int. J. Environ. Res. Public Health 18:8017. doi: 10.3390/ijerph18158017

Wang, D. X. M., Yao, J., Zirek, Y., Reijnierse, E. M., and Maier, A. B. (2020). Muscle mass, strength, and physical performance predicting activities of daily living: a meta-analysis. J. Cachexia Sarcopenia Muscle 11, 3-25. doi: 10.1002/jcsm. 12502

Watson, T. L., and Blanchard-Fields, F. (1998). Thinking with your head and your heart: age differences in everyday problem-solving strategy preferences. Aging Neuropsychol. Cognit. 5, 225-240. doi: 10.1076/ anec.5.3.225.613

White, S. M., Wójcicki, T. R., and McAuley, E. (2009). Physical activity and quality of life in community dwelling older adults. Health Qual. Life Outcomes 7, 1-7. doi: 10.1186/1477-7525-7-10

WHO (1997). WHOQOL: Measuring Quality of Life. World Health Organization. Available at: https://www.who.int/mental_health/media/68.pdf

WHO (2020a). Ageing: healthy ageing and functional ability. Available at: https:// www.who.int/westernpacific/news/q-a-detail/ageing-healthy-ageing-andfunctional-ability (Accessed April 17, 2021).

WHO (2020b). Decade of healthy ageing. Available at: https://www.who.int/ initiatives/decade-of-healthy-ageing (Accessed October 26, 2020).

Williams, S. L., and French, D. P. (2011). What are the most effective intervention techniques for changing physical activity self-efficacy and physical activity behaviour--and are they the same? Health Educ. Res. 26, 308-322. doi: 10.1093/her/cyr005

Wilson, J. M., Lee, J., and Shook, N. J. (2020). COVID-19 worries and mental health: the moderating effect of age. Aging Ment. Health 25, 1289-1296. doi: 10.1080/13607863.2020.1856778

Windle, G., Bennett, K. M., and Noyes, J. (2011). A methodological review of resilience measurement scales. Health Qual. Life Outcomes 9:8. doi: 10.1186/1477-7525-9-8

Windle, G., Hughes, D., Linck, P., Russell, I., and Woods, B. (2010). Is exercise effective in promoting mental well-being in older age? A systematic review. Aging Ment. Health 14, 652-669. doi: 10.1080/13607861003713232 
Windsor, T. D. (2009). Persistence in goal striving and positive reappraisal as psychosocial resources for ageing well: a dyadic analysis. Aging Ment. Health 13, 874-884. doi: 10.1080/13607860902918199

Winningham, R. G., and Pike, N. L. (2007). A cognitive intervention to enhance institutionalized older adults' social support networks and decrease loneliness. Aging Ment. Health 11, 716-721. doi: 10.1080/13607860701366228

Wrzus, C., Hänel, M., Wagner, J., and Neyer, F. J. (2013). Social network changes and life events across the life span: a meta-analysis. Psychol. Bull. 139, 53-80. doi: 10.1037/a0028601

Conflict of Interest: The authors declare that the research was conducted in the absence of any commercial or financial relationships that could be construed as a potential conflict of interest.
Publisher's Note: All claims expressed in this article are solely those of the authors and do not necessarily represent those of their affiliated organizations, or those of the publisher, the editors and the reviewers. Any product that may be evaluated in this article, or claim that may be made by its manufacturer, is not guaranteed or endorsed by the publisher.

Copyright (c) 2021 Brinkhof, Huth, Murre, de Wit, Krugers and Ridderinkhof. This is an open-access article distributed under the terms of the Creative Commons Attribution License (CC BY). The use, distribution or reproduction in other forums is permitted, provided the original author(s) and the copyright owner(s) are credited and that the original publication in this journal is cited, in accordance with accepted academic practice. No use, distribution or reproduction is permitted which does not comply with these terms. 